\title{
Hyperplanes of Hermitian dual polar spaces of rank 3 containing a quad
}

\author{
Bart De Bruyn \\ Ghent University, Department of Mathematics, Krijgslaan 281 (S22), B-9000 Gent, Belgium, \\ E-mail: bdb@cage.ugent.be
}

\begin{abstract}
Let $\mathbb{F}$ and $\mathbb{F}^{\prime}$ be two fields such that $\mathbb{F}^{\prime}$ is a quadratic Galois extension of $\mathbb{F}$. If $|\mathbb{F}| \geq 3$, then we provide sufficient conditions for a hyperplane of the Hermitian dual polar space $D H\left(5, \mathbb{F}^{\prime}\right)$ to arise from the Grassmann embedding. We use this to give an alternative proof for the fact that all hyperplanes of $D H\left(5, q^{2}\right), q \neq 2$, arise from the Grassmann embedding, and to show that every hyperplane of $D H\left(5, \mathbb{F}^{\prime}\right)$ that contains a quad $Q$ is either classical or the extension of a non-classical ovoid of $Q$. We will also give a classification of the hyperplanes of $D H\left(5, \mathbb{F}^{\prime}\right)$ that contain a quad and arise from the Grassmann embedding.
\end{abstract}

Keywords: Hermitian dual polar space, hyperplane, Grassmann embedding, quad MSC2010: 51A45, 51A50

\section{Introduction}

\subsection{Motivation and framework}

In this subsection, we briefly discuss the wider framework in which the results of the present paper should be seen. The detailed discussion of the main results is postponed till Section 1.3. These results are rather technical and several definitions and notations need to be given in order to fully understand them. The relevant notions and notations will be introduced in Section 1.2.

This paper is about hyperplanes of Hermitian dual polar spaces of rank 3. A hyperplane of a general point-line geometry $\mathcal{S}$ is a set of points meeting each line in either a singleton or the whole line. If $\mathcal{S}$ admits a full projective embedding, then there is a standard way to construct hyperplanes of $\mathcal{S}$, namely by intersecting an embedded copy of $\mathcal{S}$ with a hyperplane of the ambient projective space. Hyperplanes that can be obtained in this way are said to be classical. A number of problems now naturally arise:

- For a given point-line geometry $\mathcal{S}$, classify all its hyperplanes, or if this goal is too ambitious, classify all hyperplanes under suitable additional restrictions. 
- For a given fully embeddable point-line geometry $\mathcal{S}$, determine whether all its hyperplanes are classical. If that is not the case, is it then possible to give (necessary and) sufficient conditions that guarantee that a hyperplane is classical?

The above problems have already been considered in the literature for several point-line geometries. In the present paper, we consider these problems for so-called Hermitian dual polar spaces of rank 3 .

All hyperplanes of finite Hermitian dual polar spaces of rank 3 have been classified in $[9,10]$. For infinite dual polar spaces however, it seems not possible to classify all hyperplanes due to the possibility to construct hyperplanes via a process that invokes transfinite recursion. In the present paper, we will be able to obtain a classification of the hyperplanes under the additional restriction that there are deep quads, see Theorems 1.2 and 1.3 and the accompanying discussion.

With exception of $D H(5,4)$, all classical hyperplanes of Hermitian dual polar spaces of rank 3 must arise from the so-called Grassmann embedding ([8, Corollary 1.4(ii)]). In the present paper, we also give sufficient conditions for hyperplanes of rank 3 Hermitian dual polar spaces to arise from that embedding, see Theorem 1.1. This technical theorem can be used to give an alternative proof for the fact that all hyperplanes of finite Hermitian dual polar spaces of rank 3 are classical (a result originally obtained in [10]). In fact, also the classification of all hyperplanes containing a deep quad will rely on that result.

\subsection{Definitions and notations}

Throughout this paper, $\mathbb{F}$ and $\mathbb{F}^{\prime}$ denote two fields such that $\mathbb{F}^{\prime}$ is a quadratic Galois extension of $\mathbb{F}$, and $\psi$ denotes the unique nontrivial element of the Galois group $\operatorname{Gal}\left(\mathbb{F}^{\prime} / \mathbb{F}\right)$. For every $n \geq 2$, let $V_{n}^{\prime}$ be an $n$-dimensional vector space over $\mathbb{F}^{\prime}$. After having chosen a fixed basis $B_{n}$ in $V_{n}^{\prime}$, the set $V_{n}$ of all $\mathbb{F}$-linear combinations of the elements of $B_{n}$ can naturally be regarded as an $n$-dimensional vector space over $\mathbb{F}$. For every vector $\bar{v}=\sum_{\bar{b} \in B_{n}} \lambda_{\bar{b}} \cdot \bar{b}$ of $V_{n}^{\prime}$, we define $\bar{v}^{\psi}:=\sum_{\bar{b} \in B_{n}} \lambda_{\bar{b}}^{\psi} \cdot \bar{b}$. Clearly, $\bar{v}^{\psi}=\bar{v}$ if and only if $\bar{v} \in V_{n}$.

If $L$ is a line of $\mathrm{PG}\left(V_{n}^{\prime}\right)$ through two distinct points $\left\langle\bar{v}_{1}\right\rangle$ and $\left\langle\bar{v}_{2}\right\rangle$ of $\mathrm{PG}\left(V_{n}^{\prime}\right)$, then the set $\left\{\left\langle\lambda_{1} \bar{v}_{1}+\lambda_{2} \bar{v}_{2}\right\rangle \mid\left(\lambda_{1}, \lambda_{2}\right) \in \mathbb{F}^{2} \backslash\{(0,0)\}\right\}$ is a so-called Baer-F-F-subline of $L$. Suppose $\mathcal{H}$ is Hermitian variety of $\mathrm{PG}\left(V_{n}^{\prime}\right)$ defined by a $\psi$-Hermitian form of $V_{n}^{\prime}$. Then any line $L$ of $\mathrm{PG}\left(V_{n}^{\prime}\right)$ not contained in $\mathcal{H}$ that contains at least two points of $\mathcal{H}$ will intersect $\mathcal{H}$ in a Baer- $\mathbb{F}$-subline of $L$. We call such a line of $\mathrm{PG}\left(V_{n}^{\prime}\right)$ a hyperbolic line of $\mathcal{H}$.

Let $n \in \mathbb{N} \backslash\{0,1\}$. Then let $f_{n}^{\prime}$ denote a nondegenerate alternating bilinear form on $V_{2 n}^{\prime}$ such that $f_{n}^{\prime}(\bar{x}, \bar{y}) \in \mathbb{F}$ for all $\bar{x}, \bar{y} \in V_{2 n}$. The form $f_{n}^{\prime}$ induces a nondegenerate alternating bilinear form $f_{n}$ on $V_{2 n}$. For all $\bar{x}, \bar{y} \in V_{2 n}^{\prime}$, we put $h_{n}(\bar{x}, \bar{y}):=f_{n}^{\prime}\left(\bar{x}, \bar{y}^{\psi}\right)$. Then $h_{n}^{\prime}$ is a nondegenerate skew-Hermitian form on $V_{2 n}^{\prime}$. The set of all points $\langle\bar{x}\rangle$ of $\operatorname{PG}\left(V_{2 n}^{\prime}\right)$ for which $h_{n}(\bar{x}, \bar{x})=0$ is a nonsingular Hermitian variety $H\left(2 n-1, \mathbb{F}^{\prime}\right)$ of Witt index $n$ of $\mathrm{PG}\left(V_{2 n}^{\prime}\right)$ which contains all points of $\mathrm{PG}\left(V_{2 n}\right)$. We denote by $D H\left(2 n-1, \mathbb{F}^{\prime}\right)$ the corresponding Hermitian dual polar space of rank $n$. The points of $D H\left(2 n-1, \mathbb{F}^{\prime}\right)$ are the subspaces of dimension $n-1$ of $H\left(2 n-1, \mathbb{F}^{\prime}\right)$ and the lines of $D H\left(2 n-1, \mathbb{F}^{\prime}\right)$ are certain subsets of such $(n-1)$-dimensional subspaces, with incidence being containment. Specifically, there exists a bijective correspondence between the lines of $D H\left(2 n-1, \mathbb{F}^{\prime}\right)$ 
and the $(n-2)$-dimensional subspaces of $H\left(2 n-1, \mathbb{F}^{\prime}\right)$, with each line of $D H\left(2 n-1, \mathbb{F}^{\prime}\right)$ consisting of all the $(n-1)$-dimensional subspaces of $H\left(2 n-1, \mathbb{F}^{\prime}\right)$ containing a given $(n-2)$-dimensional subspace of $H\left(2 n-1, \mathbb{F}^{\prime}\right)$. The isomorphism class of $H\left(2 n-1, \mathbb{F}^{\prime}\right)$ and $D H\left(2 n-1, \mathbb{F}^{\prime}\right)$ are not necessarily uniquely determined by $n$ and $\mathbb{F}^{\prime}$, but can also depend on the subfield $\mathbb{F}$ of $\mathbb{F}^{\prime}$. In the finite case, we have $\mathbb{F} \cong \mathbb{F}_{q}$ and $\mathbb{F}^{\prime} \cong \mathbb{F}_{q^{2}}$ for some prime power $q$, and $H\left(2 n-1, \mathbb{F}^{\prime}\right)$ and $D H\left(2 n-1, \mathbb{F}^{\prime}\right)$ are then also denoted by $H\left(2 n-1, q^{2}\right)$ and $D H\left(2 n-1, q^{2}\right)$, respectively. Notice that if $L$ is a line of $\mathrm{PG}\left(V_{2 n}^{\prime}\right)$, then $L \cap H\left(2 n-1, \mathbb{F}^{\prime}\right)$ is either empty, a singleton, the whole point set of $L$ or a Baer- $\mathbb{F}$-subline of $L$. In the latter case, $L$ is a hyperbolic line of $H\left(2 n-1, \mathbb{F}^{\prime}\right)$. The dual polar space $D H\left(3, \mathbb{F}^{\prime}\right)$ is a generalized quadrangle $(\mathrm{GQ})$ isomorphic to the GQ $Q(5, \mathbb{F})$ defined by the points and lines lying on a nonsingular quadric of Witt index 2 of $\mathrm{PG}(5, \mathbb{F})$ which becomes a quadric of Witt index 3 over the extension field $\mathbb{F}^{\prime}$ of $\mathbb{F}$.

Distances $\mathrm{d}(\cdot, \cdot)$ in the dual polar space $D H\left(2 n-1, \mathbb{F}^{\prime}\right)$ will always be measured in its collinearity graph. The maximal distance between two points of $D H\left(2 n-1, \mathbb{F}^{\prime}\right)$ is equal to $n$, in which case the two points are said to be opposite. The dual polar space $D H\left(2 n-1, \mathbb{F}^{\prime}\right)$ is an example of a near polygon, meaning that for every point $x$ and every line $L$, there exists a unique point on $L$ nearest to $x$. If $x$ is a point of $D H\left(2 n-1, \mathbb{F}^{\prime}\right)$ and $i \in \mathbb{N}$, then $\Gamma_{i}(x)$ denotes the set of all points at distance $i$ from $x$. If $i \in \mathbb{N}$ and $X$ is a nonempty set of points of $D H\left(2 n-1, \mathbb{F}^{\prime}\right)$, then $\Gamma_{i}(X)$ denotes the set of points at distance $i$ from $X$, i.e. the set of all points $y$ for which $\min \{\mathrm{d}(y, x) \mid x \in X\}=i$.

If $x_{1}$ and $x_{2}$ are two points of $D H\left(5, \mathbb{F}^{\prime}\right)$ at distance 2 from each other, then the smallest convex subspace $Q\left(x_{1}, x_{2}\right)$ of $D H\left(5, \mathbb{F}^{\prime}\right)$ containing $x_{1}$ and $x_{2}$ is called a quad. There exists a bijective correspondence between the quads $Q$ of $D H\left(5, \mathbb{F}^{\prime}\right)$ and the points $x_{Q}$ of $H\left(5, \mathbb{F}^{\prime}\right)$ : the quad $Q$ consists of all planes of $H\left(5, \mathbb{F}^{\prime}\right)$ that contain the point $x_{Q}$. The points and lines of $D H\left(5, \mathbb{F}^{\prime}\right)$ that are contained in a given quad $Q$ define a point-line geometry $\widetilde{Q}$ isomorphic to $D H\left(3, \mathbb{F}^{\prime}\right) \cong Q(5, \mathbb{F})$. If $L_{1}$ and $L_{2}$ are two distinct lines through the same point, then $L_{1}$ and $L_{2}$ are contained in a unique quad, which we will denote by $Q\left(L_{1}, L_{2}\right)$. Any two distinct quads of $D H\left(5, \mathbb{F}^{\prime}\right)$ through the same point intersect in a line. If $Q$ is a quad of $D H\left(5, \mathbb{F}^{\prime}\right)$ and $x$ is a point not contained in $Q$, then $x$ is collinear with a unique point $\pi_{Q}(x)$ of $Q$. If $Q_{1}$ and $Q_{2}$ are two disjoint quads of $D H\left(5, \mathbb{F}^{\prime}\right)$, then the map $Q_{1} \rightarrow Q_{2} ; x \mapsto \pi_{Q_{2}}(x)$ defines an isomorphism between $\widetilde{Q_{1}}$ and $\widetilde{Q_{2}}$.

A hyperplane of $D H\left(2 n-1, \mathbb{F}^{\prime}\right)$ is a set $H$ of points, distinct from the whole point set, such that each line has either one or all of its points in $H$. A set $\Pi$ of hyperplanes of $D H\left(2 n-1, \mathbb{F}^{\prime}\right)$ is called a pencil of hyperplanes if every point is contained in either 1 or all elements of $\Pi$. Every hyperplane of $Q(5, \mathbb{F})$ is either an ovoid, a singular hyperplane or a $Q(4, \mathbb{F})$-subquadrangle (see also Section 3.1). Here, an ovoid is a set of points having a unique point in common with each line, a singular hyperplane consists of all points collinear with or equal to a given point, and a $Q(4, \mathbb{F})$-subquadrangle is a subquadrangle on which the induced geometry is isomorphic to the GQ $Q(4, \mathbb{F})$ defined by the points and lines lying on a nonsingular quadric of Witt index 2 of $\mathrm{PG}(4, \mathbb{F})$. Ovoids in $Q(5, \mathbb{F})$ can only exist if $\mathbb{F}$ is infinite. If $Q$ is a quad of $D H\left(5, \mathbb{F}^{\prime}\right)$ and $\sigma$ is a hyperplane of $\widetilde{Q} \cong Q(5, \mathbb{F})$, then $Q \cup\left\{x \in \Gamma_{1}(Q) \mid \pi_{Q}(x) \in \sigma\right\}$ is a hyperplane of $D H\left(5, \mathbb{F}^{\prime}\right)$, called the extension of $\sigma$.

A full projective embedding of $D H\left(2 n-1, \mathbb{F}^{\prime}\right)$ is an injective mapping $\epsilon$ from its point- 
set $\mathcal{P}_{n}$ to the set of points of a projective space $\Sigma$ satisfying: (i) $\left\langle\epsilon\left(\mathcal{P}_{n}\right)\right\rangle_{\Sigma}=\Sigma$; (ii) $\epsilon$ maps every line of $D H\left(2 n-1, \mathbb{F}^{\prime}\right)$ to a (full) line of $\Sigma$. If $\epsilon: D H\left(2 n-1, \mathbb{F}^{\prime}\right) \rightarrow \Sigma$ is a full projective embedding of $D H\left(2 n-1, \mathbb{F}^{\prime}\right)$ and $U$ is a hyperplane of $\Sigma$, then $H_{U}:=\epsilon^{-1}\left(\epsilon\left(\mathcal{P}_{n}\right) \cap U\right)$ is a hyperplane of $D H\left(2 n-1, \mathbb{F}^{\prime}\right)$. We say that the hyperplane $H_{U}$ arises from the embedding $\epsilon$. Since hyperplanes of thick dual polar spaces are maximal proper subspaces ([1, Theorem 7.3], [17, Lemma 6.1]), we must have $U=\left\langle\epsilon\left(H_{U}\right)\right\rangle_{\Sigma}$, and so there exists a bijective correspondence between the hyperplanes of $\Sigma$ and the hyperplanes of $D H\left(2 n-1, \mathbb{F}^{\prime}\right)$ arising from $\epsilon$. A hyperplane of $D H\left(2 n-1, \mathbb{F}^{\prime}\right)$ is called classical if it arises from some projective embedding. By [3, Proposition 5.1] and [4, Proposition 5.2], the dual polar space $D H\left(2 n-1, \mathbb{F}^{\prime}\right)$ admits a nice full projective embedding in $\operatorname{PG}\left(\left(\begin{array}{c}2 n \\ n\end{array}\right)-1, \mathbb{F}\right)$, the so-called Grassmann embedding of $\operatorname{DH}\left(2 n-1, \mathbb{F}^{\prime}\right)$.

If $L$ is a hyperbolic line of $H\left(5, \mathbb{F}^{\prime}\right)$, then we denote by $\Omega_{L}$ the set of all quads $Q$ of $D H\left(5, \mathbb{F}^{\prime}\right)$ for which $x_{Q} \in L$. The set $\Omega_{L}$ is a set of mutually disjoint quads satisfying the following properties:

(H1) every line of $\operatorname{DH}\left(5, \mathbb{F}^{\prime}\right)$ meeting two distinct quads of $\Omega_{L}$ meets every quad of $\Omega_{L}$;

(H2) $M=\bigcup_{Q \in \Omega_{L}}(Q \cap M)$ for every line $M$ of $D H\left(5, \mathbb{F}^{\prime}\right)$ meeting all quads of $\Omega_{L}$.

The set $\Omega_{L}$ is called a hyperbolic set of quads of $D H\left(5, \mathbb{F}^{\prime}\right)$. Every two disjoint quads $Q_{1}$ and $Q_{2}$ of $D H\left(5, \mathbb{F}^{\prime}\right)$ are contained in a unique hyperbolic set of quads which we denote by $\Omega\left(Q_{1}, Q_{2}\right)$.

\subsection{The main results}

By [10] and [15, Corollary 2, p. 180], we know that all hyperplanes of the finite Hermitian dual polar space $D H\left(5, q^{2}\right)$ are classical, and that they even all arise from the Grassmann embedding if $q \geq 3$. These conclusions are no longer valid in the infinite case, due to the possibility to construct hyperplanes by means of transfinite recursion. The following theorem will be useful to show that certain hyperplanes of $D H\left(5, \mathbb{F}^{\prime}\right)$ arise from the Grassmann embedding. We will prove it in Section 4.

Theorem 1.1 Suppose $|\mathbb{F}| \geq 3$. Let $\Omega$ be a hyperbolic set of quads of $D H\left(5, \mathbb{F}^{\prime}\right)$ and let $\mathcal{Q}$ be the set of all quads of $D H\left(5, \mathbb{F}^{\prime}\right)$ which either belong to $\Omega$ or intersect each quad of $\Omega$ in a line. For every $Q \in \mathcal{Q}$, let $\mathcal{C}_{Q}$ be a set of classical hyperplanes of $\widetilde{Q}$ such that the following hold:

(1) If $Q_{1}$ and $Q_{2}$ are two disjoint quads of $\mathcal{Q}$, then $\pi_{Q_{2}}\left(\sigma_{1}\right) \in \mathcal{C}_{Q_{2}}$ for every $\sigma_{1} \in \mathcal{C}_{Q_{1}}$.

(2) If $Q \in \mathcal{Q}$ and $\sigma_{1}, \sigma_{2}$ are two distinct elements of $\mathcal{C}_{Q}$, then there exists a unique pencil $\Pi$ of classical hyperplanes of $\widetilde{Q}$ such that $\sigma_{1}, \sigma_{2} \in \Pi$.

Suppose $H$ is a hyperplane of $D H\left(5, \mathbb{F}^{\prime}\right)$ such that $H \cap Q \in \mathcal{C}_{Q}$ for every quad $Q \in \mathcal{Q}$ not contained in $H$. Then $H$ arises from the Grassmann embedding of $D H\left(5, \mathbb{F}^{\prime}\right)$. 
We will now discuss two applications of Theorem 1.1.

Application 1. Suppose $\mathbb{F}=\mathbb{F}_{q}$ with $q \geq 2$ a prime power. Let $\Omega$ and $\mathcal{Q}$ be sets of quads of $D H\left(5, q^{2}\right)$ as in Theorem 1.1. For every quad $Q \in \mathcal{Q}$, let $\mathcal{C}_{Q}$ denote the set of all hyperplanes of $\widetilde{Q}$. Note that since $\widetilde{Q} \cong Q(5, q)$ does not have ovoids $([14,1.8 .3]$ or $[16$, Theorem $5.1])$, all its hyperplanes are either singular hyperplanes or $Q(4, q)$-subquadrangles and hence classical. The condition (1) of Theorem 1.1 is trivially fulfilled. Condition (2) is satisfied by Lemma 4.4 of [10]. So, Theorem 1.1 implies that all hyperplanes of $D H\left(5, q^{2}\right)$, $q \geq 3$, arise from the Grassmann embedding. Observe also that the condition $|\mathbb{F}| \geq 3$ in Theorem 1.1 cannot be omitted, since $D H(5,4)$ has hyperplanes not arising from the Grassmann embedding, see [9].

The proof of Theorem 1.1 that we will give thus offers an alternative proof for the fact that all hyperplanes of $D H\left(5, q^{2}\right), q \geq 3$, arise from the Grassmann embedding. This result was originally proved in [10], where it was obtained after a lengthy treatment (also yielding a complete classification of the hyperplanes) that relied on some classification results of certain sets of points in $\operatorname{PG}\left(2, q^{2}\right)$ due to Tallini-Scafati $[18,19]$ and a characterization result for classical unitals independently obtained by Faina \& Korchmáros [11] and Lefèvre-Percsy [13].

Application 2. Suppose $|\mathbb{F}| \geq 3$. Consider two disjoint quads $Q_{1}$ and $Q_{2}$ of $D H\left(5, \mathbb{F}^{\prime}\right)$ and put $\Omega:=\Omega\left(Q_{1}, Q_{2}\right)$. Let $\mathcal{Q}$ be as in Theorem 1.1 and let $\sigma_{1}$ be a given classical hyperplane of $\widetilde{Q_{1}}$. For every quad $Q \in \Omega$, define $\mathcal{C}_{Q}:=\left\{\pi_{Q}\left(\sigma_{1}\right)\right\}$, and for every quad $Q$ meeting $Q_{1}$ and $Q_{2}$ in lines, let $\mathcal{C}_{Q}$ denote the set of (classical) hyperplanes of $\widetilde{Q}$ containing $Q \cap Q_{2}$. Then the condition (1) of Theorem 1.1 is fulfilled. In Lemma 3.5, we will show that also condition (2) is fulfilled. Theorem 1.1 thus implies that all hyperplanes $H$ of $D H\left(5, \mathbb{F}^{\prime}\right)$ for which $Q_{2} \subseteq H$ and $H \cap Q_{1}=\sigma_{1}$ arise from the Grassmann embedding.

The result mentioned at the end of Application 2 will be employed in Section 4.2 to prove the following.

Theorem 1.2 Suppose $|\mathbb{F}| \geq 3$ and $H$ is a hyperplane of $D H\left(5, \mathbb{F}^{\prime}\right)$ containing a quad $Q$. Then $H$ either arises from the Grassmann embedding of $D H\left(5, \mathbb{F}^{\prime}\right)$ or is the extension of a non-classical ovoid of $\widetilde{Q}$.

Observe that the conclusion of Theorem 1.2 does not hold if $|\mathbb{F}|=2$. Indeed, the dual polar space $D H(5,4)^{1}$ has (classical) hyperplanes containing quads that do not arise from the Grassmann embedding, see [9]. A result, similar to the one mentioned in Theorem 1.2, was obtained for symplectic dual polar spaces in [7]. In [7, Section 4.2], it was also shown that if $H$ is a hyperplane of $D W(5, \mathbb{F})$ arising from the Grassmann embedding and containing a quad, then there exists a point $x$ that is deep with respect to $H$, that means that $x^{\perp}:=\{x\} \cup \Gamma_{1}(x)$ is contained in $H$. This conclusion is no longer valid for Hermitian dual polar spaces. In [10], a class of counter examples can be found for each finite dual polar space $D H\left(5, q^{2}\right)$ (the so-called hyperplanes of Type V). These examples

\footnotetext{
${ }^{1}$ Recall that the quads of $D H(5,4)$, which are isomorphic to $Q(5,2)$, do not have ovoids.
} 
belong to the infinite family of hyperplanes of Hermitian dual polar spaces discussed in [6]. The hyperplanes of $D H\left(5, \mathbb{F}^{\prime}\right)$ belonging to this infinite family all contain a glued near hexagon of type $Q(5, \mathbb{F}) \otimes Q(5, \mathbb{F})$. For this reason, these hyperplanes of $\operatorname{DH}\left(5, \mathbb{F}^{\prime}\right)$ are said to be of glued type. In Section 5, we also prove the following.

Theorem 1.3 Suppose $H$ is a hyperplane of $D H\left(5, \mathbb{F}^{\prime}\right)$ arising from the Grassmann embedding and containing a quad. Then either $H$ contains a deep point or $H$ is of glued type.

If $H$ is a hyperplane of $D H\left(5, \mathbb{F}^{\prime}\right)$ arising from the Grassmann embedding containing a quad and a deep point, then we will also show in Section 5 that $H$ is either a singular hyperplane (consisting of all points at distance at most 2 from a given point), the extension of a classical ovoid of a quad, the extension of a $Q(4, \mathbb{F})$-subquadrangle of a quad or a certain hyperplane related to a unital of $\mathrm{PG}\left(V_{3}^{\prime}\right)$ defined by a nondegenerate $\psi$-Hermitian form of Witt index 1 of $V_{3}^{\prime}$. We discuss the latter hyperplane in more detail now.

Let $x$ and $y$ be two opposite points of $D H\left(5, \mathbb{F}^{\prime}\right)$ corresponding to the respective planes $\mathrm{PG}\left(W_{x}\right)$ and $\mathrm{PG}\left(W_{y}\right)$ of $H\left(5, \mathbb{F}^{\prime}\right)$. Let $\mathcal{U}$ denote a unital of $\mathrm{PG}\left(W_{x}\right)$ defined by a nondegenerate $\psi$-Hermitian form of Witt index 1 of $W_{x}$. For every $u \in \mathcal{U}$, there exists a unique line $L_{u}$ in $\mathrm{PG}\left(W_{y}\right)$ such that $\left\langle u, L_{u}\right\rangle$ is a plane contained in $H\left(5, \mathbb{F}^{\prime}\right)$. We denote by $\mathcal{L}^{\prime}$ the set of all lines of $\mathrm{PG}\left(W_{y}\right)$ obtained in this way, and by $\mathcal{L}$ the set of lines of $D H\left(5, \mathbb{F}^{\prime}\right)$ through $y$ corresponding to the elements of $\mathcal{L}^{\prime}$. Put $X:=x^{\perp} \cup\left(\bigcup_{L \in \mathcal{L}}\right) L$. If $\epsilon^{*}$ is the Grassmann embedding of $D H\left(5, \mathbb{F}^{\prime}\right)$ in $\Sigma^{*} \cong \operatorname{PG}(19, \mathbb{F})$, then $U:=\left\langle\epsilon^{*}(X)\right\rangle_{\Sigma^{*}}$ is a hyperplane of $\Sigma^{*}$ (see Lemma 3.10 and Proposition 3.13) and $H_{U}$ is a hyperplane of $D H\left(5, \mathbb{F}^{\prime}\right)$ having $x$ as deep point. The lines through $y$ contained in $H_{U}$ are precisely the lines of $\mathcal{L}$. The quads through $x$ contained in $H_{U}$ are precisely the quads meeting a line of $\mathcal{L}$, or equivalently, the quads $Q$ for which $x_{Q} \in \mathcal{U}$.

\section{Some generation problems}

If $X$ is a set of points of a point-line geometry $\mathcal{S}$, then the intersection of all subspaces of $\mathcal{S}$ containing $X$ is the smallest subspace of $\mathcal{S}$ that contains the set $X$. This subspace is denoted by $\langle X\rangle_{\mathcal{S}}$ and is called the subspace of $\mathcal{S}$ generated by $X$. If $\langle X\rangle_{\mathcal{S}}$ coincides with the whole point set of $\mathcal{S}$, then we say that $X$ generates $\mathcal{S}$ or that $X$ is a generating set of $\mathcal{S}$. If no confusion is possible, we will also write $\langle X\rangle$ instead of $\langle X\rangle_{\mathcal{S}}$. The aim of this section is to determine generating sets of certain geometries related to Hermitian varieties. These generating sets will play a crucial role in the proof of Theorem 1.1.

\subsection{Generation problems for some geometries related to unitals}

We continue with the notation of Section 1. Consider the projective plane $\mathrm{PG}\left(V_{3}^{\prime}\right)$. Recall that if $\bar{v}_{1}$ and $\bar{v}_{2}$ are two linearly independent vectors of $V_{3}^{\prime}$, then $\left\{\left\langle\lambda_{1} \bar{v}_{1}+\lambda_{2} \bar{v}_{2}\right\rangle \mid\left(\lambda_{1}, \lambda_{2}\right) \in\right.$ $\left.\mathbb{F}^{2} \backslash\{(0,0)\}\right\}$ is a Baer- $\mathbb{F}$-subline of the line of $\mathrm{PG}\left(V_{3}^{\prime}\right)$ corresponding to $\left\langle\bar{v}_{1}, \bar{v}_{2}\right\rangle$. In this section, we will use the notion Baer subline as an abbreviation of Baer- $\mathbb{F}$-subline. Observe 
that any two distinct Baer sublines of a given line of $\mathrm{PG}\left(V_{3}^{\prime}\right)$ intersect in at most two points. A set $\mathcal{L}$ of lines through a given point $p$ of $\mathrm{PG}\left(V_{3}^{\prime}\right)$ is called a Baer pencil with center $p$ if for some line (and hence all lines) $M$ not containing $p$, the set $\bigcup_{L \in \mathcal{L}} M \cap L$ is a Baer subline of $M$.

Suppose $\mathcal{U}$ is a unital (that means a nonempty nonsingular Hermitian curve) of $\mathrm{PG}\left(V_{3}^{\prime}\right)$ defined by a nondegenerate skew- $\psi$-Hermitian form $h$ of Witt index 1 of $V_{3}^{\prime}$. If $L$ is a hyperbolic line of $\mathcal{U}$, then $\bar{L}$ denotes the Baer subline $L \cap \mathcal{U}$ of $L$. If $L$ is a hyperbolic line of $\mathcal{U}$ and $p$ is a point of $\mathcal{U}$ not belonging to $L$, then $\operatorname{BP}(p, L)$ denotes the Baer pencil consisting of all lines that join $p$ with a point of $\bar{L}$.

Lemma 2.1 Let $L$ be a line of $\mathrm{PG}\left(V_{3}^{\prime}\right)$ and $x_{1}, x_{2}, \ldots, x_{k}$ a collection of $k \in \mathbb{N}$ mutually distinct points of $L$. If $\mathbb{F}$ is infinite then $L \backslash\left\{x_{1}, x_{2}, \ldots, x_{k}\right\}$ cannot be covered by a finite number of Baer sublines. If $\mathbb{F} \cong \mathbb{F}_{q}$ for some prime power $q$, then the number of Baer sublines necessary to cover $L \backslash\left\{x_{1}, x_{2}, \ldots, x_{k}\right\}$ is at least $\frac{q^{2}+1-k}{q+1}$.

Proof. If $\mathbb{F}=\mathbb{F}_{q}$ for some prime power $q$, then the number of Baer sublines necessary to cover $A:=L \backslash\left\{x_{1}, x_{2}, \ldots, x_{k}\right\}$ is at least $\frac{|A|}{q+1}=\frac{q^{2}+1-k}{q+1}$.

Suppose now that $\mathbb{F}$ is infinite and that $A:=L \backslash\left\{x_{1}, x_{2}, \ldots, x_{k}\right\}$ is covered by the elements of a finite set $\mathcal{B}$ of Baer sublines. Take two distinct points $x$ and $y$ of $A$. Then $x$ and $y$ are contained in exactly $|\mathbb{F}|+1$ Baer sublines and hence there exists a Baer subline $B$ through $x$ and $y$ not belonging to $\mathcal{B}$. Notice that $\left|B \cap B^{\prime}\right| \leq 2$ for every $B^{\prime} \in \mathcal{B}$. If $A$ would be covered by the elements of $\mathcal{B}$, then also $A^{\prime}:=B \backslash\left\{x_{1}, x_{2}, \ldots, x_{k}\right\}$ would be covered by the elements of $\mathcal{B}$, and so we would have $\left|A^{\prime}\right| \leq 2 \cdot|\mathcal{B}|$, which is impossible as $A^{\prime}$ is infinite and $\mathcal{B}$ is finite.

The following is an immediate consequence of Lemma 2.1.

Corollary 2.2 Let $p$ be a point of $\mathrm{PG}\left(V_{3}^{\prime}\right)$, let $\mathcal{L}_{p}$ denote the set of all lines through $p$ and let $L_{1}, L_{2}, \ldots, L_{k}$ be a collection of $k \in \mathbb{N}$ mutually distinct elements of $\mathcal{L}_{p}$. If $\mathbb{F}$ is infinite, then $\mathcal{L}_{p} \backslash\left\{L_{1}, L_{2}, \ldots, L_{k}\right\}$ cannot be covered by a finite number of Baer pencils with center $p$. If $\mathbb{F} \cong \mathbb{F}_{q}$ for some prime power $q$, then the number of Baer pencils with center $p$ necessary to cover $\mathcal{L}_{p} \backslash\left\{L_{1}, L_{2}, \ldots, L_{k}\right\}$ is at least $\frac{q^{2}+1-k}{q+1}$.

Lemma 2.3 Let $p_{1}, p_{2}$ and $p_{3}$ be three points of $\mathcal{U}$ not on the same line. Then every point $p \in \overline{p_{1} p_{3}} \backslash\left\{p_{3}\right\}$ is contained in a unique hyperbolic line $M$ distinct from $p p_{3}=p_{1} p_{3}$ for which $\operatorname{BP}\left(p_{3}, M\right)=\operatorname{BP}\left(p_{3}, p_{1} p_{2}\right)$.

Proof. We can choose an ordered basis $\left(\bar{e}_{1}, \bar{e}_{2}, \bar{e}_{3}\right)$ in $V_{3}^{\prime}$ such that $p_{1}=\left\langle\bar{e}_{1}\right\rangle, p_{2}=\left\langle\bar{e}_{2}\right\rangle$, $p_{3}=\left\langle\bar{e}_{3}\right\rangle$ and such that the matrix describing $h$ with respect to $\left(\bar{e}_{1}, \bar{e}_{2}, \bar{e}_{3}\right)$ is equal to

$$
\left[\begin{array}{ccc}
0 & 1 & 1 \\
-1 & 0 & \lambda \\
-1 & -\lambda^{\psi} & 0
\end{array}\right]
$$

where $\lambda \in \mathbb{F}^{\prime}$. Since $h$ is nondegenerate, the determinant $\lambda^{\psi}-\lambda$ of this matrix should be nonzero, implying that $\lambda \in \mathbb{F}^{\prime} \backslash \mathbb{F}$. Suppose $p=\left\langle\bar{e}_{1}+l_{1} \bar{e}_{3}\right\rangle$ for some $l_{1} \in \mathbb{F}^{\prime}$. Then 
$l_{1} \in \mathbb{F}$ since $p \in \mathcal{U}$. If $M$ is a hyperbolic line through $p$ distinct from $p p_{3}=p_{1} p_{3}$ such that $\operatorname{BP}\left(p_{3}, M\right)=\operatorname{BP}\left(p_{3}, p_{1} p_{2}\right)$, then $M \cap p_{2} p_{3} \in \mathcal{U} \backslash\left\{p_{3}\right\}$. Now, a general point $r$ of $\overline{p_{2} p_{3}} \backslash\left\{p_{3}\right\}$ has the form $\left\langle\bar{e}_{2}+\frac{l_{2}}{\lambda \psi} \bar{e}_{3}\right\rangle$ where $l_{2} \in \mathbb{F}$. A general point $r^{\prime}$ of $\overline{p_{1} p_{2}} \backslash\left\{p_{2}\right\}$ has the form $\left\langle\bar{e}_{1}+k \bar{e}_{2}\right\rangle$ where $k \in \mathbb{F}$. The intersection of the lines $p_{3} r^{\prime}$ and $p r$ is the point $\left\langle\bar{e}_{1}+k \bar{e}_{2}+\left(l_{1}+\frac{k l_{2}}{\lambda^{\psi}}\right) \bar{e}_{3}\right\rangle$. It follows that the line $M=p r$ satisfies the required condition if and only if

$$
h\left(\bar{e}_{1}+k \bar{e}_{2}+\left(l_{1}+\frac{k l_{2}}{\lambda^{\psi}}\right) \bar{e}_{3}, \bar{e}_{1}+k \bar{e}_{2}+\left(l_{1}+\frac{k l_{2}}{\lambda^{\psi}}\right) \bar{e}_{3}\right)=0
$$

for all $k \in \mathbb{F}$. The latter equation is equivalent with

$$
\frac{k l_{2}\left(\lambda^{\psi}-\lambda\right)}{\lambda^{\psi+1}}+k l_{1}\left(\lambda-\lambda^{\psi}\right)=0
$$

So, we see that there is only one possibility for $l_{2}$, namely $l_{2}=l_{1} \lambda^{\psi+1}$. So, $r$ and $M=p r$ are uniquely determined.

The following is an immediate consequence of Lemma 2.3.

Corollary 2.4 Suppose $p_{1}$ and $p_{2}$ are two distinct points of $\mathcal{U}$. Then there cannot exist two hyperbolic lines $K$ and $L$ through $p_{2}$ such that $K, L, p_{2} p_{1}$ are mutually distinct and $\operatorname{BP}\left(p_{1}, K\right)=\operatorname{BP}\left(p_{1}, L\right)$.

Lemma 2.5 Let $p_{1}, p_{2}$ and $p_{3}$ be three points of $\mathcal{U}$ not on the same line, let $T_{i}, i \in$ $\{1,2,3\}$, denote the unique line through $p_{i}$ tangent to $\mathcal{U}$, and let $\mathcal{L}^{*}$ denote the unique Baer pencil with center $p_{3}$ containing $p_{3} p_{1}, p_{3} p_{2}$ and $T_{3}$. Then $\operatorname{BP}\left(p_{3}, p p_{2}\right) \cap \operatorname{BP}\left(p_{3}, p^{\prime} p_{2}\right)$ $=\left\{p_{3} p_{1}, p_{3} p_{2}\right\}$ for any two distinct points $p, p^{\prime}$ of $A:=\overline{p_{1} p_{3}} \backslash\left\{p_{3}\right\}$. Moreover, the set $\bigcup_{p \in A} \operatorname{BP}\left(p_{3}, p p_{2}\right)$ consists of all lines through $p_{3}$, except for those contained in $\mathcal{L}^{*} \backslash$ $\left\{p_{3} p_{1}, p_{3} p_{2}\right\}$.

Proof. Clearly, $\left\{p_{3} p_{1}, p_{3} p_{2}\right\}$ is a subset of $\operatorname{BP}\left(p_{3}, p p_{2}\right) \cap \operatorname{BP}\left(p_{3}, p^{\prime} p_{2}\right)$. If $\mid \operatorname{BP}\left(p_{3}, p p_{2}\right) \cap$ $\operatorname{BP}\left(p_{3}, p^{\prime} p_{2}\right) \mid \geq 3$, then we would have $\operatorname{BP}\left(p_{3}, p p_{2}\right)=\operatorname{BP}\left(p_{3}, p^{\prime} p_{2}\right)$, in contradiction with Corollary 2.4. Hence, $\operatorname{BP}\left(p_{3}, p p_{2}\right) \cap \operatorname{BP}\left(p_{3}, p^{\prime} p_{2}\right)=\left\{p_{3} p_{1}, p_{3} p_{2}\right\}$.

As before, we can choose a basis $\left(\bar{e}_{1}, \bar{e}_{2}, \bar{e}_{3}\right)$ in $V_{3}^{\prime}$ such that $p_{1}=\left\langle\bar{e}_{1}\right\rangle, p_{2}=\left\langle\bar{e}_{2}\right\rangle$, $p_{3}=\left\langle\bar{e}_{3}\right\rangle$ and such that the matrix describing $h$ with respect to $\left(\bar{e}_{1}, \bar{e}_{2}, \bar{e}_{3}\right)$ is equal to

$$
\left[\begin{array}{ccc}
0 & 1 & 1 \\
-1 & 0 & \lambda \\
-1 & -\lambda^{\psi} & 0
\end{array}\right]
$$

where $\lambda \in \mathbb{F}^{\prime} \backslash \mathbb{F}$ (as $h$ is nondegenerate). The tangent line $T_{3}$ through the point $p_{3}$ is equal to $\left\langle\bar{e}_{3},-\lambda \bar{e}_{1}+\bar{e}_{2}\right\rangle$. So, $\mathcal{L}^{*}$ consists of all lines connecting $p_{3}$ with a point of the form $\left\langle\mu_{1} \bar{e}_{1}+\mu_{2} \lambda^{\psi} \bar{e}_{2}\right\rangle$ where $\left(\mu_{1}, \mu_{2}\right) \in \mathbb{F}^{2} \backslash\{(0,0)\}$.

Now, a point of $A$ has the form $\left\langle\bar{e}_{1}+k \bar{e}_{3}\right\rangle$ with $k \in \mathbb{F}$. The points of $\mathcal{U} \backslash\left\{p_{2}\right\}$ on the line through $\left\langle\bar{e}_{1}+k \bar{e}_{3}\right\rangle(k \in \mathbb{F})$ and $\left\langle\bar{e}_{2}\right\rangle$ are of the form $\left\langle\bar{e}_{1}+k \bar{e}_{3}+\left(k^{\prime}-k k^{\prime} \lambda^{\psi}\right) \bar{e}_{2}\right\rangle$ where 
$k^{\prime} \in \mathbb{F}$. Now, $k^{\prime}-k k^{\prime} \lambda^{\psi}$ can take all values in $\mathbb{F}^{\prime}$ except those of the form $k^{\prime \prime} \lambda^{\psi}$ with $k^{\prime \prime} \in \mathbb{F} \backslash\{0\}$. The validity of the lemma is now easily seen.

Definition. If $X \mp \mathcal{U}$, then $\mathcal{S}_{X}^{\prime}$ denotes the point-line geometry with point set $\mathcal{U} \backslash X$ whose lines are all the hyperbolic lines of $\mathcal{U}$ disjoint from $X$ (natural incidence).

Lemma 2.6 Suppose $|\mathbb{F}| \geq 3$. Let $L$ be a hyperbolic line of $\mathcal{U}$ and let $p_{1}, p_{2}, p_{3}$ be three points of $\mathcal{U} \backslash L$ not on the same line such that $p_{3} p_{1} \cap L$ and $p_{3} p_{2} \cap L$ are not contained in $\mathcal{U}$. Then $\left\{p_{1}, p_{2}, p_{3}\right\}$ is a generating set of the point-line geometry $\mathcal{S}_{\bar{L}}^{\prime}$.

Proof. The proof we present here only works if $|\mathbb{F}| \geq 9$. With the aid of the computer algebra system GAP [12], we have however verified that the result also holds if $\mathbb{F}=\mathbb{F}_{q}$ with $q \in\{3,4,5,7,8\}$. The code we have used to verify this can be found in the appendix at the end of this paper.

Since $p_{2} p_{3} \in \mathrm{BP}\left(p_{2}, p_{1} p_{3}\right)$ and $p_{2} p_{3} \notin \mathrm{BP}\left(p_{2}, L\right)$, we have $\left|\mathrm{BP}\left(p_{2}, p_{1} p_{3}\right) \cap \mathrm{BP}\left(p_{2}, L\right)\right| \leq 2$. Let $A_{1}$ denote the set of all points $p$ of $p_{1} p_{3}$ for which $p p_{2} \in \operatorname{BP}\left(p_{2}, p_{1} p_{3}\right) \cap \operatorname{BP}\left(p_{2}, L\right)$. Then $\left|A_{1}\right| \leq 2$ and $A_{1} \subseteq A:=\overline{p_{1} p_{3}} \backslash\left\{p_{3}\right\}$. Let $\mathcal{L}_{1}^{*}$ denote the unique Baer pencil with center $p_{3}$ containing $p_{3} p_{1}, p_{3} p_{2}$ and the unique line through $p_{3}$ tangent to $\mathcal{U}$. Put $\mathcal{L}_{2}^{*}:=\operatorname{BP}\left(p_{3}, L\right)$.

For every $p \in A$, put $\mathcal{L}_{p}:=B\left(p_{3}, p p_{2}\right)$. If $p \in A \backslash A_{1}$, then all points of $\overline{p p_{2}}$ belong to the subspace $\left\langle p_{1}, p_{2}, p_{3}\right\rangle$ of $\mathcal{S}_{\bar{L}}^{\prime}$ generated by $\left\{p_{1}, p_{2}, p_{3}\right\}$. If $p \in A \backslash A_{1}$ and $r \in \overline{p p_{2}}$ such that $p_{3} r \notin \mathcal{L}_{2}^{*}$, then also all points of $\overline{p_{3} r}$ belong to $\left\langle p_{1}, p_{2}, p_{3}\right\rangle$.

By Lemma 2.5, it follows that all points of $\mathcal{U} \backslash L$ are contained in $\left\langle p_{1}, p_{2}, p_{3}\right\rangle$, except possibly those contained in a line of $\mathcal{L}_{1}^{*} \cup \mathcal{L}_{2}^{*} \cup\left(\bigcup_{a \in A_{1}} \mathcal{L}_{a}\right)$.

Now, suppose $p$ is a point of $\mathcal{U} \backslash L$ contained on a line of $\mathcal{L}_{1}^{*} \cup \mathcal{L}_{2}^{*} \cup\left(\bigcup_{a \in A_{1}} \mathcal{L}_{a}\right)$, but not on $p_{3} p_{1} \cup p_{3} p_{2}$. Since $\left|A_{1}\right| \leq 2$ and $|\mathbb{F}| \geq 9$, the number of (nontangent) lines through $p_{3}$ not contained in $\mathcal{L}_{1}^{*} \cup \mathcal{L}_{2}^{*} \cup\left(\bigcup_{a \in A_{1}} \mathcal{L}_{a}\right)$ is at least 2 by Corollary 2.2. So, by Corollary 2.4, there exists a hyperbolic line $M$ through $p_{3}$ not contained in $\mathcal{L}_{1}^{*} \cup \mathcal{L}_{2}^{*} \cup\left(\bigcup_{a \in A_{1}} \mathcal{L}_{a}\right)$ such that $\mathrm{BP}(p, M) \neq \mathrm{BP}(p, L)$. So, $|\mathrm{BP}(p, M) \cap \mathrm{BP}(p, L)| \leq 2$ and there exists a point $p^{\prime} \in \bar{M} \backslash\left\{p_{3}\right\}$ such that the line $p p^{\prime}$ does not belong to $\operatorname{BP}(p, L)$. Since $M=p_{3} p^{\prime}$ is not contained in $\mathcal{L}_{1}^{*} \cup \mathcal{L}_{2}^{*} \cup\left(\bigcup_{a \in A_{1}} \mathcal{L}_{a}\right)$, the Baer pencil $\operatorname{BP}\left(p_{3}, p^{\prime} p\right)$ intersects the set $\mathcal{L}_{1}^{*} \cup \mathcal{L}_{2}^{*} \cup\left(\bigcup_{a \in A_{1}} \mathcal{L}_{a}\right)$ in at most 8 elements. Since $|\mathbb{F}|+1 \geq 10$, the Baer subline $\overline{p^{\prime} p}$ of $p^{\prime} p$ contains at least two elements of $\left\langle p_{1}, p_{2}, p_{3}\right\rangle$. Since $p^{\prime} p \cap L$ is not contained in $\mathcal{U}$, the point $p$ should be contained in $\left\langle p_{1}, p_{2}, p_{3}\right\rangle$.

Lemma 2.7 Suppose $|\mathbb{F}| \geq 3$. Let $L$ be a hyperbolic line of $\mathcal{U}$ and let $p_{1}, p_{2}, p_{3}$ be three mutually distinct points of $\mathcal{U} \backslash L$ such that $p_{3} p_{1} \neq p_{3} p_{2}$ and $p_{1} p_{3} \cap L$ is not contained in $\mathcal{U}$. Then $\left(\overline{p_{3} p_{1}} \cup \overline{p_{3} p_{2}}\right) \backslash L$ generates the point-line geometry $\mathcal{S}_{\bar{L}}^{\prime}$.

Proof. Put $\mathcal{L}_{1}:=\mathrm{BP}\left(p_{1}, p_{2} p_{3}\right)$ and $\mathcal{L}_{2}:=\mathrm{BP}\left(p_{1}, L\right)$. Since $p_{1} p_{3} \in \mathcal{L}_{1} \backslash \mathcal{L}_{2}$, we have $\left|\mathcal{L}_{1} \cap \mathcal{L}_{2}\right| \leq 2$ and so there exists a $K \in \mathcal{L}_{1} \backslash \mathcal{L}_{2}$ distinct from $p_{1} p_{3}$. Put $\left\{p_{2}^{\prime}\right\}:=K \cap p_{2} p_{3}$. Since $p_{1} p_{3}$ and $p_{1} p_{2}^{\prime}$ are disjoint from $\bar{L}$, Lemma 2.6 implies that $\left\{p_{1}, p_{2}^{\prime}, p_{3}\right\}$ generates the point-line geometry $\mathcal{S}_{\bar{L}}^{\prime}$. Hence, also $\left(\overline{p_{3} p_{1}} \cup \overline{p_{3} p_{2}}\right) \backslash L$ generates $\mathcal{S}_{\bar{L}}^{\prime}$. 
Proposition 2.8 Suppose $|\mathbb{F}| \geq 3$. Let $X \subseteq \mathcal{U}$ be empty or a singleton. If $p_{1}, p_{2}, p_{3}$ are three points of $\mathcal{U} \backslash X$ not on the same line, then $\left\{p_{1}, p_{2}, p_{3}\right\}$ generates $\mathcal{S}_{X}^{\prime}$.

Proof. Let $p^{*}$ be a point of $\mathcal{U} \backslash\left\{p_{1}, p_{2}, p_{3}\right\}$ such that $X=\left\{p^{*}\right\}$ if $|X|=1$. Since $p_{1} p_{2} \cap p_{1} p_{3} \cap p_{2} p_{3}=\emptyset$, we may without loss of generality suppose that $p^{*} \notin p_{3} p_{1} \cup p_{3} p_{2}$. Put $\mathcal{L}_{i}:=B\left(p^{*}, p_{i} p_{3}\right), i \in\{1,2\}$. By Corollary 2.2 and the fact that $|\mathbb{F}| \neq 2$, there exists a hyperbolic line $L$ through $p^{*}$ not contained in $\mathcal{L}_{1} \cup \mathcal{L}_{2}$. By Lemma 2.6, it follows that every point of $\mathcal{U} \backslash L$ is contained in $\left\langle p_{1}, p_{2}, p_{3}\right\rangle$. Now, every point $p$ of $L \backslash X$ is contained in a hyperbolic line $L^{\prime}$ distinct from $L$ and every point of $L^{\prime} \backslash\{p\}$ belongs to $\left\langle p_{1}, p_{2}, p_{3}\right\rangle$, showing that also $p$ belongs to $\left\langle p_{1}, p_{2}, p_{3}\right\rangle$.

Proposition 2.9 Suppose $|\mathbb{F}| \geq 3$. Let $L$ be a hyperbolic line of $\mathcal{U}$ and let $p_{1}, p_{2}, p_{3}$ be three points of $\mathcal{U} \backslash L$ not on the same line. Then $\left(\overline{p_{3} p_{1}} \cup \overline{p_{3} p_{2}}\right) \backslash L$ generates the point-line geometry $\mathcal{S}_{\bar{L}}^{\prime}$.

Proof. Let $p_{i}^{\prime}, i \in\{1,2\}$, denote the unique point in the intersection of the lines $p_{3} p_{i}$ and $L$. If at least one of $p_{1}^{\prime}, p_{2}^{\prime}$ does not belong to $\mathcal{U}$, then the claim follows from Lemma 2.7 . So, we will suppose that $p_{1}^{\prime}$ and $p_{2}^{\prime}$ belong to $\mathcal{U}$. Corollary 2.4 implies that $\operatorname{BP}\left(p_{1}, p_{2} p_{3}\right) \neq$ $\mathrm{BP}\left(p_{1}, L\right)$. Denote by $K$ an arbitrary line of $\operatorname{BP}\left(p_{1}, p_{2} p_{3}\right) \backslash \mathrm{BP}\left(p_{1}, L\right)$. Then $\bar{K}$ belongs to the subspace of $\mathcal{S}_{\bar{L}}^{\prime}$ generated by $\left(\overline{p_{3} p_{1}} \cup \overline{p_{3} p_{2}}\right) \backslash L$. As $\left(\bar{K} \cup \overline{p_{1} p_{3}}\right) \backslash L$ generates $\mathcal{S}_{\bar{L}}^{\prime}$ (Lemma $2.7)$, the set $\left(\overline{p_{3} p_{1}} \cup \overline{p_{3} p_{2}}\right) \backslash L$ should also generate $\mathcal{S}_{\bar{L}}^{\prime}$.

\subsection{Generation problems for some geometries related to $H\left(3, \mathbb{F}^{\prime}\right)$}

We continue with the notation introduced in Section 1. We put $\Sigma=\operatorname{PG}\left(V_{4}\right)$ and $\Sigma^{\prime}=$ $\mathrm{PG}\left(V_{4}^{\prime}\right)$ and we denote by $\mathcal{H}$ the Hermitian variety $H\left(3, \mathbb{F}^{\prime}\right)$ of $\Sigma^{\prime}$. Notice that $\Sigma \subseteq \mathcal{H}$. The Hermitian polarity of $\Sigma^{\prime}$ associated with $\mathcal{H}$ is denoted by $\zeta$. If $\alpha$ is a subspace of $\Sigma^{\prime}$, then we define $\bar{\alpha}:=\alpha \cap \mathcal{H}$. If $p$ is a point and $L$ is a hyperbolic line of $\mathcal{H}$ not containing $p$, then similarly as before we denote by $\operatorname{BP}(p, L)$ the Baer pencil with center $p$ obtained by joining $p$ with all points of $\bar{L}=L \cap \mathcal{H}$.

If $p=\langle\bar{x}\rangle$ is a point of $\Sigma^{\prime}$, then we define $p^{\psi}:=\left\langle\bar{x}^{\psi}\right\rangle$. We have $p=p^{\psi}$ if and only if $p \in \Sigma$. If $p \neq p^{\psi}$, then $p p^{\psi}$ is the unique line through $p$ intersecting $\Sigma$ in a Baer subline. For every set $X$ of points of $\mathrm{PG}\left(V_{4}^{\prime}\right)$, we define $X^{\psi}:=\left\{p^{\psi} \mid p \in X\right\}$. The map $p \mapsto p^{\psi}$ defines an isomorphism of $\Sigma^{\prime}$. If $\alpha$ is a subspace of $\Sigma^{\prime}$, then $\alpha \cap \alpha^{\psi}$ is a subspace of $\Sigma^{\prime}$ and $\alpha \cap \alpha^{\psi} \cap \Sigma$ is a subspace of $\Sigma$. Moreover, $\operatorname{dim}_{\Sigma^{\prime}}\left(\alpha \cap \alpha^{\psi}\right)=\operatorname{dim}_{\Sigma}\left(\alpha \cap \alpha^{\psi} \cap \Sigma\right)$.

If $\alpha$ is a plane of $\Sigma^{\prime}$, then $\alpha$ is either a tangent plane with tangent point $\alpha^{\zeta}$, or a nontangent plane intersecting $\mathcal{H}$ in a unital of $\alpha$.

Lemma 2.10 If $p \in \Sigma \subseteq \mathcal{H}$ and $\alpha=p^{\zeta}$, then $\alpha=\alpha^{\psi}$.

Proof. Put $p=\langle\bar{x}\rangle$ for some $\bar{x} \in V_{4} \backslash\{\bar{o}\}$. Since the set $U=\left\{\bar{y} \in V_{4} \mid f_{2}(\bar{x}, \bar{y})=0\right\}$ is a hyperplane of $V_{4}$, it generates a hyperplane $U^{\prime}$ of $V_{4}^{\prime}$ which corresponds to a plane $\alpha$ of $\Sigma^{\prime}$ for which $\alpha=\alpha^{\psi}$. Since $h_{2}(\bar{y}, \bar{x})=f_{2}^{\prime}(\bar{y}, \bar{x})=0$ for all $\bar{y} \in U^{\prime}$, we have that $\alpha=p^{\zeta}$.

Lemma 2.11 If $p \in \mathcal{H} \backslash \Sigma$, then $p p^{\psi}$ is contained in $\mathcal{H}$ and hence the tangent plane $p^{\zeta}$ contains $p p^{\psi}$. 
Proof. Put $p=\langle\bar{x}\rangle$, where $\bar{x} \in V_{4}^{\prime} \backslash\{\bar{o}\}$. Since $h_{2}(\bar{x}, \bar{x})=0, h_{2}\left(\bar{x}, \bar{x}^{\psi}\right)=f_{2}^{\prime}(\bar{x}, \bar{x})=0$ and $h_{2}\left(\bar{x}^{\psi}, \bar{x}^{\psi}\right)=f_{2}^{\prime}\left(\bar{x}^{\psi}, \bar{x}\right)=-f_{2}^{\prime}\left(\bar{x}, \bar{x}^{\psi}\right)=-h_{2}(\bar{x}, \bar{x})=0$, we have that $h_{2}\left(\mu_{1} \bar{x}+\mu_{2} \bar{x}^{\psi}, \mu_{1} \bar{x}+\right.$ $\left.\mu_{2} \bar{x}^{\psi}\right)=0$ for all $\mu_{1}, \mu_{2} \in \mathbb{F}^{\prime}$. Hence, the line $p p^{\psi}$ is contained in $\mathcal{H}$.

Lemma 2.12 One of the following cases occurs for a plane $\alpha$ of $\Sigma^{\prime}$ :

(1) $\alpha=\alpha^{\psi}$ and $\alpha$ is a tangent plane whose tangent point belongs to $\Sigma$;

(2) $\alpha \cap \alpha^{\psi}$ is a hyperbolic line $L_{\alpha}, \alpha$ is a nontangent plane and $\alpha \cap \Sigma$ is a Baer subline of $L_{\alpha}$ equal to $L_{\alpha} \cap \mathcal{H}$;

(3) $\alpha \cap \alpha^{\psi}$ is a line $L_{\alpha}$ which is contained in $\mathcal{H}, \alpha$ is a tangent plane whose tangent point $p$ belongs to $L_{\alpha} \backslash \Sigma, L_{\alpha}=p p^{\psi}$ and $\alpha \cap \Sigma$ is a Baer subline of $L_{\alpha}$.

Proof. Either $\alpha=\alpha^{\psi}$ or $\alpha \cap \alpha^{\psi}$ is a line $L_{\alpha}$.

(1) Suppose first that $\alpha=\alpha^{\psi}$. Then $\alpha$ is generated by three vectors $\bar{v}_{1}, \bar{v}_{2}$ and $\bar{v}_{3}$ of $V_{4}$. Let $U$ denote the 3 -space of $V_{4}$ generated by $\bar{v}_{1}, \bar{v}_{2}$ and $\bar{v}_{3}$. Then there exists a nonzero vector $\bar{v}^{*} \in U$ (uniquely determined, up to a nonzero factor) such that $f_{2}\left(\bar{v}^{*}, \bar{u}\right)=0$, $\forall \bar{u} \in U$. Since $h_{2}\left(\bar{u}, \bar{v}^{*}\right)=f_{2}\left(\bar{u}, \bar{v}^{*}\right)=0, \forall \bar{u} \in U$, we have that $\alpha$ must be a tangent plane whose tangent point $\left\langle\bar{v}^{*}\right\rangle$ belongs to $\Sigma$.

(2) Suppose that $\alpha \cap \alpha^{\psi}$ is a line $L_{\alpha}$. Then $L_{\alpha}$ is generated by two points of $V_{4}$ and hence $L_{\alpha} \cap \Sigma$ is a Baer subline of $L_{\alpha}$. Since every point of $\alpha \cap \Sigma$ is also contained in $\alpha^{\psi}$, we have that $\alpha \cap \Sigma=L_{\alpha} \cap \Sigma$. Notice also that $\alpha \cap \Sigma=L_{\alpha} \cap \Sigma \subseteq L_{\alpha} \cap \mathcal{H}$. If $\alpha$ is a nontangent plane, then $L_{\alpha}$ cannot be contained in $\mathcal{H}$, and so we must then have that $\alpha \cap \Sigma=L_{\alpha} \cap \Sigma=L_{\alpha} \cap \mathcal{H}$. Suppose now that $\alpha$ is a tangent plane with tangent point $p=\alpha^{\zeta}$. By Lemma 2.10, $p \notin \Sigma$. Lemma 2.11 then implies that $p p^{\psi} \subseteq \alpha$. Hence, $p p^{\psi}=\left(p p^{\psi}\right)^{\psi} \subseteq \alpha^{\psi}$ and $p p^{\psi} \subseteq \alpha \cap \alpha^{\psi}=L_{\alpha}$, i.e. $L_{\alpha}=p p^{\psi}$. By Lemma 2.11, $L_{\alpha}=p p^{\zeta}$ is contained in $\mathcal{H}$.

Definition. If $X \mp \mathcal{H}$, then we denote by $\mathcal{S}_{X}$ the point-line geometry whose points are the elements of $\mathcal{H} \backslash X$ and whose lines are the hyperbolic lines of $\mathcal{H}$ disjoint from $X$ (natural incidence). The geometry $\mathcal{S}_{\emptyset}$ is called the geometry of the hyperbolic lines of $\mathcal{H}$.

Proposition 2.13 Suppose $|\mathbb{F}| \geq 3$. Then the point-line geometry $\mathcal{S}_{\emptyset}$ can be generated by four points.

Let $\alpha$ be a nontangent plane of $\Sigma^{\prime}$, and put $\mathcal{U}:=\bar{\alpha}=\alpha \cap \mathcal{H}$. Let $p_{1}, p_{2}$ and $p_{3}$ be three distinct points of $\mathcal{U}$ not contained in the same line. Then $\left\langle p_{1}, p_{2}, p_{3}\right\rangle$ consists of all points of $\mathcal{U}$ by Proposition 2.8. Now, let $M$ denote a hyperbolic line through $p_{3}$ not contained in $\alpha$, and let $p_{4}$ be any point of $\bar{M} \backslash\left\{p_{3}\right\}$. We show that $\left\langle p_{1}, p_{2}, p_{3}, p_{4}\right\rangle=\mathcal{H}$. Note that $\bar{M} \subseteq\left\langle p_{1}, p_{2}, p_{3}, p_{4}\right\rangle$.

There are $|\mathbb{F}|+1$ tangent planes through $M$, namely the $|\mathbb{F}|+1$ planes $\langle M, x\rangle$, where $x \in \overline{M^{\zeta}}$. Denote these $|\mathbb{F}|+1$ tangent planes by $\alpha_{i}, i \in I$, for some index set $I$ of size $|\mathbb{F}|+1$. We denote by $\alpha^{*}$ the unique plane through $M$ intersecting $\alpha$ in a line that has only the point $p_{3}$ in common with $\mathcal{U}$. Then $\alpha^{*} \notin\left\{\alpha_{i} \mid i \in I\right\}$. If $\beta$ is a (nontangent) plane 
through $M$ distinct from $\alpha^{*}$ and all $\alpha_{i}$ 's, then $\langle\bar{M} \cup \overline{(\beta \cap \alpha)}\rangle=\bar{\beta}$ by Proposition 2.8. So, every point of $\mathcal{H}$ not contained in $\left(\bigcup_{i \in I} \alpha_{i}\right) \cup \alpha^{*}$ belongs to $\left\langle p_{1}, p_{2}, p_{3}, p_{4}\right\rangle$.

Suppose $x$ is a point of $\left(\bigcup_{i \in I} \overline{\alpha_{i}}\right) \cup \overline{\alpha^{*}}$ not contained in $M \cup M^{\zeta}$. Then there exists a point $x^{\prime} \in \bar{M}$ such that $x x^{\prime}$ is a hyperbolic line. Let $\beta$ be a nontangent plane through $x x^{\prime}$ that is distinct from $\alpha^{*}$ if $x \in \alpha^{*}$. Then $M$ is not contained in $\beta$ and $\left\{\beta \cap \alpha_{i} \mid i \in I\right\}$ is a Baer pencil of $\beta$ with center $x^{\prime}$. There exist two hyperbolic lines $M_{1}$ and $M_{2}$ through $x^{\prime}$ contained in $\beta$ not belonging to this Baer pencil and also distinct from the line $\beta \cap \alpha^{*}$. As $\overline{M_{1}} \cup \overline{M_{2}}$ is contained in $\left\langle p_{1}, p_{2}, p_{3}, p_{4}\right\rangle$, we must have that $\bar{\beta} \subseteq\left\langle p_{1}, p_{2}, p_{3}, p_{4}\right\rangle$ by Proposition 2.8. In particular, we have $x \in\left\langle p_{1}, p_{2}, p_{3}, p_{4}\right\rangle$.

Now, let $x$ be a point of $\overline{M^{\zeta}}$ and consider a hyperbolic line $M^{\prime}$ through $x$ distinct from $M^{\zeta}$. As $\overline{M^{\prime}} \backslash\{x\}$ is contained in $\left\langle p_{1}, p_{2}, p_{3}, p_{4}\right\rangle$, we must have that $x$ itself is also contained in $\left\langle p_{1}, p_{2}, p_{3}, p_{4}\right\rangle$. We conclude that every point of $\mathcal{H}$ belongs to $\left\langle p_{1}, p_{2}, p_{3}, p_{4}\right\rangle$.

We wish to notice that Proposition 2.13 is not new. This result was already proved (in a different way) in [8, Lemma 3.3]. However, the arguments given above will be recycled in the proofs of the following two propositions.

Proposition 2.14 Suppose $|\mathbb{F}| \geq 3$. If $L$ is a totally isotropic line of $\mathcal{H}$, then $\mathcal{S}_{L}$ can be generated by four points.

Proof. Let $\alpha$ be a nontangent plane of $\Sigma^{\prime}$. Then $\alpha$ has precisely one point in common with $L$. Put $\mathcal{U}:=\bar{\alpha}$. Let $p_{1}, p_{2}$ and $p_{3}$ be three distinct points of $\mathcal{U} \backslash L$ not contained on the same line. Then $\left\langle p_{1}, p_{2}, p_{3}\right\rangle$ consists of all points of $\mathcal{U} \backslash L$ by Proposition 2.8. Now, let $M$ denote a hyperbolic line through $p_{3}$ not contained in $\alpha \cup\left\langle p_{3}, L\right\rangle$ and let $p_{4}$ be any point of $\bar{M} \backslash\left\{p_{3}\right\}$. We show that $\left\langle p_{1}, p_{2}, p_{3}, p_{4}\right\rangle=\mathcal{H}$. Note that $\bar{M} \subseteq\left\langle p_{1}, p_{2}, p_{3}, p_{4}\right\rangle$.

There are $|\mathbb{F}|+1$ tangent planes through $M$, namely the $|\mathbb{F}|+1$ planes $\langle M, x\rangle$, where $x \in \overline{M \zeta}$. We denote these $|\mathbb{F}|+1$ tangent planes by $\alpha_{i}, i \in I$, for some index set $I$ of size $|\mathbb{F}|+1$. We denote by $\alpha^{*}$ the unique plane through $M$ intersecting $\alpha$ in a line that has only the point $p_{3}$ in common with $\mathcal{U}$. Then $\alpha^{*} \notin\left\{\alpha_{i} \mid i \in I\right\}$. If $\beta$ is a (nontangent) plane through $M$ distinct from $\alpha^{*}$ and all $\alpha_{i}$ 's, then $\beta$ intersects $L$ in precisely one point and hence $\langle(\bar{M} \cup \overline{(\beta \cap \alpha)}) \backslash L\rangle=\bar{\beta} \backslash L$ by Proposition 2.8. So, every point of $\mathcal{H} \backslash L$ not contained in $\left(\bigcup_{i \in I} \alpha_{i}\right) \cup \alpha^{*}$ belongs to $\left\langle p_{1}, p_{2}, p_{3}, p_{4}\right\rangle$.

Suppose $x$ is a point of $\left(\bigcup_{i \in I} \overline{\alpha_{i}}\right) \cup \overline{\alpha^{*}}$, not contained in $M \cup M^{\zeta} \cup L$. Then there exists a point $x^{\prime} \in \bar{M}$ such that $x x^{\prime}$ is a hyperbolic line. Let $\beta$ be a nontangent plane through $x x^{\prime}$ that is distinct from $\alpha^{*}$ if $x \in \alpha^{*}$. Then $M$ is not contained in $\beta$ and $\left\{\beta \cap \alpha_{i} \mid i \in I\right\}$ is a Baer pencil of $\beta$ with center $x^{\prime}$. There exist two hyperbolic lines $M_{1}$ and $M_{2}$ through $x^{\prime}$ contained in $\beta$ not belonging to this Baer pencil and also distinct from the line $\beta \cap \alpha^{*}$. As $\left(\overline{M_{1}} \cup \overline{M_{2}}\right) \backslash L$ is contained in $\left\langle p_{1}, p_{2}, p_{3}, p_{4}\right\rangle$, we must have $\bar{\beta} \backslash L \subseteq\left\langle p_{1}, p_{2}, p_{3}, p_{4}\right\rangle$ by Proposition 2.8. In particular, we have $x \in\left\langle p_{1}, p_{2}, p_{3}, p_{4}\right\rangle$.

Finally, let $x$ be a point of $\overline{M^{\zeta}} \backslash L$ and consider a hyperbolic line $M^{\prime}$ through $x$ distinct from $M^{\zeta}$ and not meeting $L$. As $\overline{M^{\prime}} \backslash\{x\}$ is contained in $\left\langle p_{1}, p_{2}, p_{3}, p_{4}\right\rangle$, we must have 
that $x$ itself is also contained in $\left\langle p_{1}, p_{2}, p_{3}, p_{4}\right\rangle$. We conclude that every point of $\mathcal{H} \backslash L$ belongs to $\left\langle p_{1}, p_{2}, p_{3}, p_{4}\right\rangle$.

Proposition 2.15 Suppose $|\mathbb{F}| \geq 3$. Then the point-line geometry $\mathcal{S}_{\Sigma}$ can be generated by four points.

Proof. Let $\alpha$ be a nontangent plane of $\Sigma^{\prime}$. By Lemma 2.12, $L_{\alpha}=\alpha \cap \alpha^{\psi}$ is a line and $L_{\alpha} \cap \mathcal{H}$ is a Baer subline of $L_{\alpha}$ equal to $\alpha \cap \Sigma$. Put $\mathcal{U}:=\bar{\alpha}=\alpha \cap \mathcal{H}$. Let $p_{1}, p_{2}$ and $p_{3}$ be three points of $\mathcal{U} \backslash L_{\alpha}$ not contained on the same line such that $p_{3} p_{1}$ and $p_{3} p_{2}$ do not belong to $\operatorname{BP}\left(p_{3}, L_{\alpha}\right)$. Then $\left\langle p_{1}, p_{2}, p_{3}\right\rangle$ consists of all points of $\mathcal{U} \backslash L_{\alpha}$ by Lemma 2.6.

As $p_{3} \notin \Sigma$, we have $p_{3}^{\psi} \neq p_{3}$. The line $p_{3} p_{3}^{\psi}$ is contained in $\mathcal{H}$ by Lemma 2.11. So, the plane $p_{3}^{\zeta}$ contains $p_{3} p_{3}^{\psi}$ and intersects $\alpha$ in a line $L^{*}$. Let $\mathcal{L}^{*}$ denote the set of lines through $p_{3}$ contained in $\mathcal{H}$. We have $p_{3} p_{3}^{\psi} \in \mathcal{L}^{*}$ and $L^{*} \notin \mathcal{L}^{*}$.

Let $\alpha^{\prime}$ be a nontangent plane through $p_{3}$ distinct from $\alpha$. Then $\alpha^{\prime}$ intersects $\Sigma$ in a Baer subline of $L_{\alpha^{\prime}}=\alpha^{\prime} \cap\left(\alpha^{\prime}\right)^{\psi}$ such that $p_{3} \notin L_{\alpha^{\prime}}$ and hence there exists a hyperbolic line $M \subseteq \alpha^{\prime}$ through $p_{3}$ disjoint from $\Sigma$ and distinct from $\alpha \cap \alpha^{\prime}$. Let $p_{4}$ be a point of $\bar{M}$ distinct from $p_{3}$. We will show the subspace $\left\langle p_{1}, p_{2}, p_{3}, p_{4}\right\rangle$ of $\mathcal{S}_{\Sigma}$ generated by $\left\{p_{1}, p_{2}, p_{3}, p_{4}\right\}$ coincides with the whole point set $\mathcal{H} \backslash \Sigma$ of $\mathcal{S}_{\Sigma}$.

We first show that every point of $\mathcal{H} \backslash \Sigma$ that is not contained in $p_{3}^{\zeta}$ belongs to $\left\langle p_{1}, p_{2}, p_{3}, p_{4}\right\rangle$, or equivalently, that $K \backslash \Sigma$ is contained in $\left\langle p_{1}, p_{2}, p_{3}, p_{4}\right\rangle$ for every hyperbolic line $K$ through $p_{3}$. This is certainly true if $K=M$ since the hyperbolic line $M=p_{3} p_{4}$ is disjoint from $\Sigma$. Observe also that if $K$ is a hyperbolic line through $p_{3}$, then the tangent planes through $K$ are precisely the planes $\langle K, L\rangle$ where $L \in \mathcal{L}^{*}$.

Suppose $K$ is a hyperbolic line through $p_{3}$ distinct from $M$ such that the plane $\beta=$ $\langle K, M\rangle$ intersects $p_{3}^{\zeta}$ in a line that is not contained in $\mathcal{L}^{*} \cup\left\{L^{*}\right\}$. Since $\beta$ contains no line of $\mathcal{L}^{*}$, it must be a nontangent plane. Since $L^{*}$ is not contained in $\beta, \beta \cap \alpha$ must be a hyperbolic line. So, $M$ and $\beta \cap \alpha$ are two distinct hyperbolic lines through $p_{3}$. Since $\overline{\beta \cap \alpha} \backslash \Sigma$ and $\bar{M}$ are contained in $\left\langle p_{1}, p_{2}, p_{3}, p_{4}\right\rangle$, Proposition 2.9 implies that also $\bar{\beta} \backslash \Sigma$ is contained in $\left\langle p_{1}, p_{2}, p_{3}, p_{4}\right\rangle$. In particular, $\bar{K} \backslash \Sigma$ is contained in $\left\langle p_{1}, p_{2}, p_{3}, p_{4}\right\rangle$.

Suppose $K$ is a hyperbolic line through $p_{3}$ distinct from $M$ such that the plane $\beta=$ $\langle K, M\rangle$ intersects $p_{3}^{\zeta}$ in a line that is contained in $\mathcal{L}^{*} \cup\left\{L^{*}\right\}$. Let $\beta_{1}$ be a (nontangent) plane through $M$ that intersects $p_{3}^{\zeta}$ in a line $K^{\prime}$ that does not belong to $\mathcal{L}^{*} \cup\left\{L^{*}\right\}$. Then $\beta_{1}$ does not contain $K$. By the previous paragraph, we know that $\overline{\beta_{1}} \backslash \Sigma$ is contained in $\left\langle p_{1}, p_{2}, p_{3}, p_{4}\right\rangle$. Let $\beta_{2}$ be a plane through $K$ that intersects $p_{3}^{\zeta}$ in a line that does not belong to $\mathcal{L}^{*} \cup\left\{L^{*}, K^{\prime}\right\}$ and which does not contain the line $\beta_{1} \cap \alpha$. Since $\beta_{2}$ contains no line of $\mathcal{L}^{*}$, it is a nontangent plane. Since $\beta_{2}$ does not contain the line $L^{*}$, the line $\beta_{2} \cap \alpha$ is a hyperbolic line through $p_{3}$. Since $K^{\prime}$ is the unique line through $p_{3}$ contained in $\beta_{1}$ that is tangent to $\mathcal{H}$, the fact that $K^{\prime}$ is not contained in $\beta_{2}$ implies that $\beta_{1} \cap \beta_{2}$ is a hyperbolic line through $p_{3}$. If the hyperbolic lines $\beta_{1} \cap \beta_{2}$ and $\alpha \cap \beta_{2}$ would coincide, then the line $\beta_{1} \cap \alpha$ would be contained in $\beta_{2}$, which is impossible. So, $\beta_{1} \cap \beta_{2}$ and $\beta_{2} \cap \alpha$ are two distinct hyperbolic lines through $p_{3}$. Since $\overline{\beta_{1}} \backslash \Sigma$ is contained in $\left\langle p_{1}, p_{2}, p_{3}, p_{4}\right\rangle$, every point of $\overline{\beta_{1} \cap \beta_{2}} \backslash \Sigma$ belongs to $\left\langle p_{1}, p_{2}, p_{3}, p_{4}\right\rangle$. Since also every point of $\overline{\beta_{2} \cap \alpha} \backslash \Sigma$ is contained in $\left\langle p_{1}, p_{2}, p_{3}, p_{4}\right\rangle$, we have that every point of $\overline{\beta_{2}} \backslash \Sigma$ is contained in $\left\langle p_{1}, p_{2}, p_{3}, p_{4}\right\rangle$ 
by Proposition 2.9. In particular, every point of $\bar{K} \backslash \Sigma$ belongs to $\left\langle p_{1}, p_{2}, p_{3}, p_{4}\right\rangle$. We conclude that every point of $\mathcal{H} \backslash \Sigma$ that is not contained in $p_{3}^{\zeta}$ belongs to $\left\langle p_{1}, p_{2}, p_{3}, p_{4}\right\rangle$.

Now, let $x$ be an arbitrary point of $\overline{p_{3}^{\zeta}} \backslash p_{3} p_{3}^{\psi}$, and let $x^{\prime}$ be an arbitrary point of $p_{3} p_{3}^{\psi} \cap \Sigma$. Then $x^{\prime} \neq p_{3}$ and $x x^{\prime}$ is a hyperbolic line. We denote by $\gamma$ a nontangent plane through $x x^{\prime}$. Since $L_{p_{3}^{\zeta}}=p_{3} p_{3}^{\psi}$, we have $x \notin \Sigma$ and so the line $L_{\gamma}$ is distinct from $x x^{\prime}$. Hence, $L_{\gamma}$ and $x x^{\prime}$ are two distinct hyperbolic lines of $\gamma$ through the point $x^{\prime}$. Now, let $p_{3}^{\prime}$ be an arbitrary point of $\bar{\gamma} \backslash\left(L_{\gamma} \cup x x^{\prime}\right)$, and let $M_{1}^{\prime}, M_{2}^{\prime}$ be two distinct hyperbolic lines of $\gamma$ through $p_{3}^{\prime}$, not contained in $\operatorname{BP}\left(p_{3}^{\prime}, L_{\gamma}\right)$. For every $i \in\{1,2\}$, let $p_{i}^{\prime}$ be a point of $\overline{M_{i}^{\prime}} \backslash\left\{p_{3}^{\prime}\right\}$ not contained in $x x^{\prime}$. By the above, we know that $p_{1}^{\prime}, p_{2}^{\prime}$ and $p_{3}^{\prime}$ belong to $\left\langle p_{1}, p_{2}, p_{3}, p_{4}\right\rangle$. Lemma 2.6 now implies that every point of $\bar{\gamma} \backslash L_{\gamma}$ belongs to $\left\langle p_{1}^{\prime}, p_{2}^{\prime}, p_{3}^{\prime}\right\rangle$ and hence also to $\left\langle p_{1}, p_{2}, p_{3}, p_{4}\right\rangle$. We conclude that every point of $\mathcal{H} \backslash \Sigma$ that is not contained in $p_{3} p_{3}^{\psi}$ belongs to $\left\langle p_{1}, p_{2}, p_{3}, p_{4}\right\rangle$.

Now, let $x$ be an arbitrary point of $p_{3} p_{3}^{\psi} \backslash \Sigma$. Let $\alpha^{\prime}$ be a nontangent plane through $x$ and let $M$ be a hyperbolic line of $\alpha^{\prime}$ through $x$ not contained in $\operatorname{BP}\left(x, L_{\alpha^{\prime}}\right)$. Since $\Sigma \cap \alpha^{\prime}=\overline{L_{\alpha^{\prime}}}$, $M \cap \Sigma=\emptyset$. By the above, every point of $\bar{M} \backslash\{x\}$ is contained in $\left\langle p_{1}, p_{2}, p_{3}, p_{4}\right\rangle$. Hence, also the point $x$ belongs to $\left\langle p_{1}, p_{2}, p_{3}, p_{4}\right\rangle$. We conclude that every point of $\mathcal{H} \backslash \Sigma$ belongs to $\left\langle p_{1}, p_{2}, p_{3}, p_{4}\right\rangle$.

\section{Some useful results}

In this section, we prove a number of results that will be useful during the proofs of the main theorems. These results regard hyperplanes of general thick dual polar spaces of rank 3, some geometries associated with hyperplanes of $Q(5, \mathbb{F})$, pencils of hyperplanes, regular spreads of $Q(5, \mathbb{F})$ and the Grassmann embedding of $D H\left(5, \mathbb{F}^{\prime}\right)$.

\subsection{A few results regarding hyperplanes of general thick dual polar spaces of rank 3}

In this subsection, we discuss some results regarding hyperplanes of $D H\left(5, \mathbb{F}^{\prime}\right)$ that will be useful later when we prove the main results of this paper. The properties of $D H\left(5, \mathbb{F}^{\prime}\right)$ that will be relevant during the proofs of these results also hold for general thick dual polar spaces of rank 3. So, we see no reason why we should restrict our discussion here only to the dual polar space $D H\left(5, \mathbb{F}^{\prime}\right)$. Before we can state (and prove) the results, we need to give some extra definitions and properties regarding general thick dual polar spaces of rank 3 .

Suppose $\Delta$ is a thick dual polar space of rank 3. If $x$ is a point of $\Delta$, then the set $H_{x}$ of points at distance at most 2 from $x$ is a hyperplane, called the singular hyperplane with deepest point $x$. If $x$ is a point of $\Delta$, and $O$ is a set of points at distance 3 from $x$ such that every line at distance 2 from $x$ has a unique point in common with $O$, then the set $x^{\perp} \cup O$ is a hyperplane of $\Delta$, called a semi-singular hyperplane with deep point $x$.

If $H$ is a hyperplane of $\Delta$ and $Q$ is a quad, then either $Q \subseteq H$ or $Q \cap H$ is a hyperplane of $\widetilde{Q}$. If $Q \subseteq H$, then $Q$ is called a deep quad. If $Q \cap H=x^{\perp} \cap Q$ for some point $x \in Q$, 
then $Q$ is called singular (with respect to $H$ ) and $x$ is called the deep point of $Q$. The quad $Q$ is called ovoidal (respectively, subquadrangular) with respect to $H$ if $Q \cap H$ is an ovoid (respectively, a subquadrangle) of $\widetilde{Q}$. Since every hyperplane of a thick generalized quadrangle is either a singular hyperplane, an ovoid or a subquadrangle ([14, 2.3.1]), every quad is either deep, singular, ovoidal or subquadrangular with respect to $H$. If $H$ is a hyperplane of $\operatorname{DH}\left(5, \mathbb{F}^{\prime}\right)$ and $Q$ is a quad that is subquadrangular with respect to $H$, then the subquadrangle $\widetilde{Q \cap H}$ of $\widetilde{Q}$ is isomorphic to $Q(4, \mathbb{F})$. Indeed, the intersection $Q \cap H$ contains two disjoint lines and hence also the grid $G$ generated by them. Since $G$ is no hyperplane of $\widetilde{Q}$, there exists a point $x \in Q \cap H$ not contained in $G$. There exists a $Q(4, \mathbb{K})$-subquadrangle $\sigma$ of $\widetilde{Q}$ containing $G$ and $x$. Since $G$ is a maximal subspace of $\sigma$, the hyperplane $Q \cap H$ of $\widetilde{Q}$ contains $\sigma$. Since $\sigma$ itself is also a maximal subspace of $\widetilde{Q}$, we necessarily have that $Q \cap H$ coincides with $\sigma$.

The following proposition was proved in [7, Lemma 4.1] for the symplectic dual polar space $D W(5, \mathbb{F})$, but the proof given in [7] automatically extends to arbitrary thick dual polar spaces of rank 3.

Proposition 3.1 ([7]) Let $\Delta$ be a thick dual polar space of rank $3, Q$ a quad of $\Delta$ and $H$ a hyperplane containing $Q$. If every quad disjoint from $Q$ is deep or ovoidal with respect to $H$, then $H$ is the extension of an ovoid of $\widetilde{Q}$.

Proposition 3.2 Suppose $\Delta$ is a thick dual polar space of rank 3 and let $H$ be a hyperplane of $\Delta$ having a deep point $x^{*}$. Let $\mathcal{D}^{*}$ denote the set of deep quads through $x^{*}$.

(1) If $\Gamma_{3}\left(x^{*}\right) \cap H=\emptyset$, then $H$ is the singular hyperplane with deepest point $x^{*}$.

(2) If $\mathcal{D}^{*}=\emptyset$, then $H$ is a semi-singular hyperplane of $\Delta$ with deep point $x^{*}$.

(3) Suppose there exists a deep quad $Q^{*}$ through $x^{*}$ and a set $\mathcal{L}^{*}$ of lines through $x^{*}$ contained in $Q^{*}$ such that the deep quads through $x^{*}$ distinct from $Q^{*}$ are precisely the quads distinct from $Q^{*}$ containing a (necessarily unique) line of $\mathcal{L}^{*}$. Then $H$ is the extension of a hyperplane of $\widetilde{Q^{*}}$.

Proof. Note that since $\left(x^{*}\right)^{\perp} \subseteq H$, every quad through $x^{*}$ is either deep or singular with respect to $H$.

(1) The hyperplane $H_{x^{*}}$ with deepest point $x^{*}$ contains $H$ and hence equals $H$ since hyperplanes of thick dual polar spaces are maximal proper subspaces.

(2) Since there are no deep quads through $x^{*}$, we have $\Gamma_{2}\left(x^{*}\right) \cap H=\emptyset$. So, $H=\left(x^{*}\right)^{\perp} \cup O$, where $O=\Gamma_{3}\left(x^{*}\right) \cap H$. If $L$ is a line at distance 2 from $x^{*}$, then $L \cap \Gamma_{2}\left(x^{*}\right) \cap H=\emptyset$ implies that $L \cap H$ is a singleton contained in $O$. So, every line at distance 2 from $x^{*}$ meets $O$ in a singleton, showing that $H=\left(x^{*}\right)^{\perp} \cup O$ is a semi-singular hyperplane.

(3) We first show that if $x$ is a point of $Q^{*}$, then $x^{\perp} \cap H$ is either $x^{\perp}$ or $x^{\perp} \cap Q^{*}$. Obviously, this is true if $x=x^{*}$. By considering all quads through $x x^{*}$, which are either deep or 
singular with deep point $x^{*}$, we see that the claim is also valid if $x \in \Gamma_{1}\left(x^{*}\right) \cap Q^{*}$. In fact, if $x x^{*} \in \mathcal{L}^{*}$, then $x^{\perp} \cap H=x^{\perp}$. If $x x^{*} \notin \mathcal{L}^{*}$, then $x^{\perp} \cap H=x^{\perp} \cap Q^{*}$.

Suppose now that $x \in \Gamma_{2}\left(x^{*}\right)$. Suppose also that $x^{\perp} \cap H$ contains a point $u$ that is not contained in $Q^{*}$. Then the line $x u$ is contained in $H$. Let $Q$ be an arbitrary quad through $x u$, and let $v$ denote the unique point on the line $Q \cap Q^{*}$ at distance 1 from $x^{*}$. Since $v^{\perp} \cap H$ is either $v^{\perp} \cap Q^{*}$ or $v^{\perp}$, we have that $v^{\perp} \cap H \cap Q$ is either $Q \cap Q^{*}$ or $v^{\perp} \cap Q$. So, $Q$ is singular or deep. Since $x u$ and $Q \cap Q^{*}$ are two distinct lines through $x$ contained in $H$, we have that $x^{\perp} \cap Q \subseteq H$. Since $Q$ was an arbitrary quad through $x u$, we must have that $x^{\perp} \subseteq H$.

Let $\sigma$ denote the set of all points $x$ of $Q^{*}$ for which $x^{\perp} \subseteq H$. Then $H=Q^{*} \cup\{x \in$ $\left.\Gamma_{1}\left(Q^{*}\right) \mid \pi_{Q^{*}}(x) \in \sigma\right\}$. If $Q$ is a quad disjoint from $Q^{*}$, then $H \cap Q=\pi_{Q}(\sigma)$ is a hyperplane of $\widetilde{Q}$ and hence $\sigma$ must be a hyperplane of $\widetilde{Q^{*}}$, showing that $H$ is the extension of the hyperplane $\sigma$ of $\widetilde{Q^{*}}$.

Since every hyperplane of a thick generalized quadrangle is either a singular hyperplane, an ovoid or a subquadrangle, Proposition 3.2 implies the following.

Corollary 3.3 Suppose $\Delta$ is a thick dual polar space of rank 3 and let $H$ be a hyperplane of $\Delta$ having a deep point $x^{*}$. Suppose also there exists a deep quad $Q^{*}$ through $x^{*}$ and a set $\mathcal{L}^{*}$ of lines through $x^{*}$ contained in $Q^{*}$ such that the deep quads through $x^{*}$ distinct from $Q^{*}$ are precisely the quads distinct from $Q^{*}$ containing a (necessarily unique) line of $\mathcal{L}^{*}$. Then precisely one of the following cases occurs:

(1) $\mathcal{L}^{*}=\emptyset$. Then $H$ is the extension of an ovoid $O$ of $\widetilde{Q^{*}}$. Moreover, $x^{*} \in O$.

(2) $\mathcal{L}^{*}$ is a singleton $\{L\}$. Then $H$ is a singular hyperplane of $\Delta$ whose deepest point belongs to $L \backslash\left\{x^{*}\right\}$.

(3) Suppose $\left|\mathcal{L}^{*}\right| \geq 2$ and $\mathcal{L}^{*} \neq \mathcal{L}_{Q^{*}}$, where $\mathcal{L}_{Q^{*}}$ denotes the set of lines through $x^{*}$ contained in $Q^{*}$. Then there exists a subquadrangle $\sigma$ of $\widetilde{Q^{*}}$ containing $x^{*}$ such that:

- $\mathcal{L}^{*}$ consists of all lines through $x^{*}$ contained in $\sigma$;

- $\sigma$ is a hyperplane of $\widetilde{Q^{*}}$ and $H$ is the extension of $\sigma$.

(4) Suppose $\mathcal{L}^{*}=\mathcal{L}_{Q^{*}}$. Then $H$ is the singular hyperplane with deepest point $x^{*}$.

\subsection{Hyperplanes of $D H\left(3, \mathbb{F}^{\prime}\right) \cong Q(5, \mathbb{F})$ and associated geometries}

Let $\mathcal{Q}$ be a generalized quadrangle isomorphic to the generalized quadrangle $D H\left(3, \mathbb{F}^{\prime}\right) \cong$ $Q(5, \mathbb{F})$ defined in Section 1.

Suppose $L_{1}$ and $L_{2}$ are two disjoint lines of $\mathcal{Q}$. Then $L_{1}$ and $L_{2}$ are contained in a unique full subgrid $\mathcal{G}_{L_{1}, L_{2}}$. We denote by $\left\{L_{1}, L_{2}\right\}^{\perp}$ the set of all lines meeting $L_{1}$ and $L_{2}$, and by $\left\{L_{1}, L_{2}\right\}^{\perp \perp}$ the set of all lines meeting every line of $\left\{L_{1}, L_{2}\right\}^{\perp}$. Then $\left\{L_{1}, L_{2}\right\}^{\perp} \cup\left\{L_{1}, L_{2}\right\}^{\perp \perp}$ is the line set of $\mathcal{G}_{L_{1}, L_{2}}$. If $X$ is a set of points of $\mathcal{Q}$, distinct from the whole point set, then $\mathcal{A}_{X}$ denotes the following point-line geometry: 
- the points of $\mathcal{A}_{X}$ are the lines of $\mathcal{Q}$ not contained in $X$;

- the lines of $\mathcal{A}_{X}$ are are the sets $\left\{L_{1}, L_{2}\right\}^{\perp \perp}$, where $L_{1}$ and $L_{2}$ are two disjoint lines such that no line of $\left\{L_{1}, L_{2}\right\}^{\perp \perp}$ is contained in $X$;

- incidence is containment.

The geometry $\mathcal{A}_{\emptyset}$ is isomorphic to the geometry of the hyperbolic lines of $H\left(3, \mathbb{F}^{\prime}\right)$, that is, the geometry whose points are the points of $H\left(3, \mathbb{F}^{\prime}\right)$ and whose lines are the hyperbolic lines of $H\left(3, \mathbb{F}^{\prime}\right)$ (natural incidence).

We consider the three possible types of hyperplanes of $\mathcal{Q}$.

- Suppose $\sigma$ is an ovoid of $\mathcal{Q}$. Then every line of $\mathcal{Q}$ is a point of $\mathcal{A}_{\sigma}$ and every set of the form $\left\{L_{1}, L_{2}\right\}^{\perp \perp}$, with $L_{1}$ and $L_{2}$ two disjoint lines of $\mathcal{Q}$, is a line of $\mathcal{A}_{\sigma}$. The point-line geometry $\mathcal{A}_{\sigma}$ is isomorphic to the geometry of the hyperbolic lines of $H\left(3, \mathbb{F}^{\prime}\right)$.

- Suppose $\sigma$ is a singular hyperplane of $\mathcal{Q}$. There exists up to isomorphism a unique such hyperplane. If $x$ denotes the deep point of $\sigma$, then the points of $\mathcal{A}_{\sigma}$ are those lines of $\mathcal{Q}$ not containing $x$ and the lines of $\mathcal{A}_{\sigma}$ are those sets of the form $\left\{L_{1}, L_{2}\right\}^{\perp \perp}$, where $L_{1}$ and $L_{2}$ are two disjoint lines of $\mathcal{Q}$ such that $x$ is in no line of $\left\{L_{1}, L_{2}\right\}^{\perp \perp}$. The geometry $\mathcal{A}_{\sigma}$ is isomorphic to the geometry considered in Proposition 2.14.

- Suppose $\sigma$ is a $Q(4, \mathbb{F})$-subquadrangle of $\mathcal{Q} \cong Q(5, \mathbb{F})$. Up to isomorphism, there exists a unique such hyperplane. Since $Q(4, \mathbb{F}) \cong D W(3, \mathbb{F})$, every $Q(4, \mathbb{F})$-subquadrangle defines an isometric full embedding of $D W(3, \mathbb{F})$ into $D H\left(3, \mathbb{F}^{\prime}\right)$. These embeddings have been studied in [5]. From [5], it follows that the geometry $\mathcal{A}_{\sigma}$ is isomorphic to the geometry considered in Proposition 2.15.

By Propositions 2.13, 2.14 and 2.15, we thus have:

Proposition 3.4 Suppose $|\mathbb{F}| \geq 3$ and let $\sigma$ be a hyperplane of $D H\left(3, \mathbb{F}^{\prime}\right) \cong Q(5, \mathbb{F})$. Then the point-line geometry $\mathcal{A}_{\sigma}$ can be generated by a set of four points.

\subsection{Pencils of hyperplanes}

Lemma 3.5 Suppose $\sigma_{1}$ and $\sigma_{2}$ are two distinct hyperplanes of $Q(5, \mathbb{F})$ containing a line $L$. Then through every point $x$ not contained in $\sigma_{1} \cup \sigma_{2}$, there exists a unique hyperplane $\sigma_{x}$ such that $\sigma_{1} \cap \sigma_{2}=\sigma_{1} \cap \sigma_{x}=\sigma_{2} \cap \sigma_{x}$. As a consequence, $\sigma_{1}$ and $\sigma_{2}$ are contained in a unique pencil $\Pi$ of hyperplanes of $Q(5, \mathbb{F})$. Either 0,1 or all elements of $\Pi$ are singular hyperplanes. If none of the elements of $\Pi$ is a singular hyperplane, then $\sigma_{1} \cap \sigma_{2}$ is a full subgrid.

Proof. Since $\sigma_{i}, i \in\{1,2\}$, contains $L$, it is either a singular hyperplane or a $Q(4, \mathbb{F})$ subquadrangle. If $\sigma$ is a hyperplane of $Q(5, \mathbb{F})$ satisfying $\sigma_{1} \cap \sigma_{2}=\sigma_{1} \cap \sigma=\sigma_{2} \cap \sigma$, then $\sigma$ contains $L$ and hence $\sigma$ is also a singular hyperplane or a $Q(4, \mathbb{F})$-subquadrangle. Precisely one of the following three cases occurs.

(1) Suppose $\sigma_{1}$ and $\sigma_{2}$ are singular hyperplanes. Then the deep points of $\sigma_{1}$ and $\sigma_{2}$ lie on $L$ and $\sigma_{1} \cap \sigma_{2}=L$. Any hyperplane $\sigma$ satisfying $L=\sigma_{1} \cap \sigma_{2}=\sigma_{1} \cap \sigma=\sigma_{2} \cap \sigma$ 
necessarily is a singular hyperplane. So, $\sigma_{x}$ must be the singular hyperplane whose deep point is the unique point of $L$ collinear with $x$. In this case, all hyperplanes of $\Pi$ are singular hyperplanes.

(2) Suppose at least one of $\sigma_{1}, \sigma_{2}$ is a $Q(4, \mathbb{F})$-subquadrangle and $\sigma_{1} \cap \sigma_{2}$ is a full subgrid. Any hyperplane $\sigma$ satisfying $\sigma_{1} \cap \sigma_{2}=\sigma_{1} \cap \sigma=\sigma_{2} \cap \sigma$ is a $Q(4, \mathbb{F})$-subquadrangle. So, $\sigma_{x}$ must be the unique $Q(4, \mathbb{F})$-subquadrangle containing the point $x$ and the grid $\sigma_{1} \cap \sigma_{2}$. In this case, all elements of $\Pi$ are $Q(4, \mathbb{F})$-subquadrangles.

(3) Suppose at least one of $\sigma_{1}, \sigma_{2}$ is a $Q(4, \mathbb{F})$-subquadrangle, say $\sigma_{1}$, and $\sigma_{1} \cap \sigma_{2}=$ $\sigma_{1} \cap u^{\perp}$ for some point $u \in \sigma_{1}$. If $x \sim u$, then $\sigma_{x}$ must be the singular hyperplane with deep point $u$. If $x \nsim u$, then $\sigma_{x}$ must be the unique $Q(4, \mathbb{F})$-subquadrangle containing $u^{\perp} \cap \sigma_{1}$ and $x$. In this case, precisely one element of $\Pi$ is a singular hyperplane.

Let $\Omega$ be a hyperbolic set of quads of $\Delta=D H\left(5, \mathbb{F}^{\prime}\right)$. Let $\mathcal{P}_{\Omega}$ denote the set of all quads of $\Delta$ that meet each quad of $\Omega$ (necessarily in a line). If $R_{1}$ and $R_{2}$ are two disjoint elements of $\mathcal{P}_{\Omega}$, then $\Omega\left(R_{1}, R_{2}\right) \subseteq \mathcal{P}_{\Omega}$. Put $\mathcal{L}_{\Omega}:=\left\{\Omega\left(R_{1}, R_{2}\right) \mid R_{1}, R_{2} \in \mathcal{P}_{\Omega}\right.$ and $\left.R_{1} \cap R_{2}=\emptyset\right\}$ and let $\mathcal{S}_{\Omega}$ be the point-line geometry with point set $\mathcal{P}_{\Omega}$, line set $\mathcal{L}_{\Omega}$ and containment as incidence relation.

Lemma 3.6 Suppose $\Omega$ is a hyperbolic set of quads of $\Delta=D H\left(5, \mathbb{F}^{\prime}\right)$. Then:

(1) $\mathcal{S}_{\Omega}$ is isomorphic to the geometry of the hyperbolic lines of $H\left(3, \mathbb{F}^{\prime}\right)$.

(2) $\bigcup_{Q \in \mathcal{P}_{\Omega}} Q$ is the whole point set of $\Delta$. Moreover, every point of $\Delta$ not contained in $\bigcup_{Q \in \Omega} Q$ is contained in a unique element of $\mathcal{P}_{\Omega}$.

(3) If $Q_{1}$ and $Q_{2}$ are two distinct elements of $\Omega$ and if $H$ is a hyperplane of $\Delta$ such that $H \cap Q_{1}$ and $\pi_{Q_{1}}\left(H \cap Q_{2}\right)$ are distinct hyperplanes of $\widetilde{Q_{1}}$, then $\left\{\pi_{Q_{1}}(H \cap Q) \mid Q \in \Omega\right\}$ is a pencil of hyperplanes of $\widetilde{Q_{1}}$.

Proof. Consider the quad $Q_{1}$ of $\Omega$. Then $\widetilde{Q_{1}} \cong Q(5, \mathbb{F}) \cong D H\left(3, \mathbb{F}^{\prime}\right)$. If $X \varsubsetneqq Q_{1}$, then let $\mathcal{A}_{X}$ denote the point-line geometry as defined in Section 3.2. If $x \in \bigcup_{Q \in \Omega} Q$, then $L_{x}$ denotes the unique line through $x$ meeting all quads of $\Omega$.

(1) The map from $\mathcal{P}_{\Omega}$ to the set of lines of $\widetilde{Q_{1}}$ defined by $R \mapsto R \cap Q_{1}$ defines an isomorphism between the geometries $\mathcal{S}_{\Omega}$ and $\mathcal{A}_{\emptyset}$. Hence, $\mathcal{S}_{\Omega}$ is isomorphic to the geometry of the hyperbolic lines of $H\left(3, \mathbb{F}^{\prime}\right)$.

(2) If $x \in \bigcup_{Q \in \Omega} Q$, then every quad through $L_{x}$ contains $x$ and belongs to $\mathcal{P}_{\Omega}$. If $x \notin$ $\bigcup_{Q \in \Omega} Q$, then the lines $x \pi_{Q_{1}}(x)$ and $L_{\pi_{Q_{1}}(x)}$ are distinct and the quad $Q\left(x \pi_{Q_{1}}(x), L_{\pi_{Q_{1}}(x)}\right)$ is the unique quad of $\mathcal{P}_{\Omega}$ containing $x$.

(3) This follows by considering all lines meeting each quad of $\Omega$. Each such line has either one or all its points in $H$. 


\subsection{Regular spreads of $Q(5, \mathbb{F})$}

The set of all points $\langle\bar{x}\rangle \in \mathrm{PG}\left(V_{4}^{\prime}\right)$ for which $h_{2}(\bar{x}, \bar{x})=0$ is a nonsingular Hermitian variety $H\left(3, \mathbb{F}^{\prime}\right)$ of Witt index 2 and hence a GQ. The point-line dual of this GQ is isomorphic to $Q(5, \mathbb{F})$.

Now, suppose that $\theta$ is an isomorphism between $Q(5, \mathbb{F})$ and the point-line dual of $H\left(3, \mathbb{F}^{\prime}\right)$. Any two disjoint lines $L_{1}$ and $L_{2}$ of $Q(5, \mathbb{F})$ are contained in a unique full subgrid $\mathcal{G}_{L_{1}, L_{2}}$ and we denote by $\mathcal{L}_{L_{1}, L_{2}}$ the set of lines of $\mathcal{G}_{L_{1}, L_{2}}$ parallel to $L_{1}$ and $L_{2}$. Then

$$
\mathcal{L}_{L_{1}, L_{2}}^{\theta}=L_{1}^{\theta} L_{2}^{\theta} \cap H\left(3, \mathbb{F}^{\prime}\right) .
$$

A spread of $Q(5, \mathbb{F})$ is a set of lines partitioning its point set. If $S$ is a spread of $Q(5, \mathbb{F})$, then $S^{\theta}$ is an ovoid of $H\left(3, \mathbb{F}^{\prime}\right)$. If $S^{\theta}$ is a classical ovoid (i.e., is obtained by intersecting $H\left(3, \mathbb{F}^{\prime}\right)$ with a nontangent plane), then $S$ is called a classical spread of $Q(5, \mathbb{F})$. A spread $S$ of $Q(5, \mathbb{F})$ is called regular if $\mathcal{L}_{L_{1}, L_{2}} \subseteq S$ for any two distinct lines $L_{1}$ and $L_{2}$ of $S$. By $(1)$, every classical spread of $Q(5, \mathbb{F})$ is regular. Also the converse is true.

Proposition 3.7 Every regular spread of $Q(5, \mathbb{F})$ is classical.

Proof. Suppose $S$ is a regular spread of $Q(5, \mathbb{F})$. Let $L_{1}$ and $L_{2}$ be two distinct lines of $S$, put $G:=\mathcal{G}_{L_{1}, L_{2}}$ and let $\sigma$ be an arbitrary $Q(4, \mathbb{F})$-subquadrangle containing $G$. For every point $x$ of $\sigma$, let $L_{x}$ denote the unique line of $S$ containing $x$. Then $S=\mathcal{L}_{L_{1}, L_{2}} \cup\left\{L_{x} \mid x \in\right.$ $\sigma \backslash G\}$. Let $x^{*}$ be a fixed point of $\sigma \backslash G$ and put $L_{3}:=L_{x^{*}}$. For every $i \in\{1,2,3\}$, put $x_{i}:=L_{i}^{\theta}$. As $L_{3} \notin \mathcal{L}_{L_{1}, L_{2}}$, we have $x_{3} \notin x_{1} x_{2}$ and hence $\alpha=\left\langle x_{1}, x_{2}, x_{3}\right\rangle$ is a plane.

We show that $S^{\theta} \subseteq \alpha$. As $\mathcal{L}_{L_{1}, L_{2}}^{\theta}=x_{1} x_{2} \cap H\left(3, \mathbb{F}^{\prime}\right) \subseteq \alpha$, it suffices to prove that $L_{x}^{\theta} \in \alpha$ for every point $x$ of $\sigma \backslash G$. Observe that $x_{3}=L_{3}^{\theta}=L_{x^{*}}^{\theta} \in \alpha$. So, by the connectedness of $\sigma \backslash G$ it suffices to show that if $y_{1}, y_{2}$ are two distinct collinear points of $\sigma \backslash G$, then $L_{y_{1}}^{\theta} \in \alpha$ implies that $L_{y_{2}}^{\theta} \in \alpha$. Let $K$ denote the unique line of $\sigma$ through $y_{1}, y_{2}$ and let $L_{4}$ denote the unique line of $\mathcal{L}_{L_{1}, L_{2}}$ containing the unique point of $K$ belonging to $G$. Then $L_{y_{2}} \in \mathcal{L}_{L_{y_{1}}, L_{4}}$ as $S$ is regular. Since $L_{y_{1}}^{\theta}$ and $L_{4}^{\theta}$ belong to $\alpha$ and $L_{y_{2}}^{\theta} \in L_{y_{1}}^{\theta} L_{4}^{\theta}$, we have that also $L_{y_{2}}^{\theta}$ belongs to $\alpha$.

So, we have that $S^{\theta} \subseteq \alpha$. Since $S$ is a set of mutually disjoint lines, $S^{\theta}$ is a set of points that are mutually noncollinear on $H\left(3, \mathbb{F}^{\prime}\right)$. Since $S^{\theta}$ contains the Baer- $\mathbb{F}$-subline $\mathcal{L}_{L_{1}, L_{2}}^{\theta}$ and the extra point $L_{3}^{\theta}$, the plane $\alpha$ should be a nontangent plane. But then $S^{\prime}=\left(\alpha \cap H\left(3, \mathbb{F}^{\prime}\right)\right)^{\theta^{-1}}$ is a set of mutually disjoint lines of $Q\left(5, \mathbb{F}^{\prime}\right)$. Since $S \subseteq S^{\prime}$, we should have $S=S^{\prime}$, i.e. $S^{\theta}=\alpha \cap H\left(3, \mathbb{F}^{\prime}\right)$. This implies that $S$ is a classical spread.

\subsection{The Grassmann embedding of $D H\left(5, \mathbb{F}^{\prime}\right)$}

Let $\epsilon^{*}$ denote the Grassmann embedding of $\Delta=D H\left(5, \mathbb{F}^{\prime}\right)$ into $\Sigma^{*} \cong \mathrm{PG}(19, \mathbb{F})$. If $Q$ is a quad of $\Delta$, then the embedding of $\widetilde{Q} \cong Q(5, \mathbb{F})$ induced by $\epsilon^{*}$ is isomorphic to the Grassmann embedding of $\widetilde{Q} \cong Q(5, \mathbb{F})$ in $\operatorname{PG}(5, \mathbb{F})$. So, $\left\langle\epsilon^{*}(Q)\right\rangle$ is 5-dimensional. If $Q_{1}$ and $Q_{2}$ are two disjoint quads of $\Delta$, then $\left\langle\epsilon^{*}\left(Q_{1}\right)\right\rangle$ and $\left\langle\epsilon^{*}\left(Q_{2}\right)\right\rangle$ are two disjoint subspaces of $\Sigma^{*}$. 
For every hyperbolic set $\Omega$ of quads of $\Delta$, let $\mathcal{S}_{\Omega}$ denote the point-line geometry with point set $\mathcal{P}_{\Omega}$ and line set $\mathcal{L}_{\Omega}$ as defined in Section 3.3. If $|\mathbb{F}| \geq 3$, then by Proposition 2.13 and Lemma 3.6(1), $\mathcal{S}_{\Omega}$ can be generated by four points.

Lemma 3.8 Suppose $|\mathbb{F}| \geq 3$ and $Q_{1}, Q_{2}$ are two disjoint quads of $D H\left(5, \mathbb{F}^{\prime}\right)$. Let $x_{1}, x_{2}, \ldots, x_{6}$ be six points of $Q_{1}$ such that $\left\langle\epsilon^{*}\left(x_{1}\right), \epsilon^{*}\left(x_{2}\right), \ldots, \epsilon^{*}\left(x_{6}\right)\right\rangle=\left\langle\epsilon^{*}\left(Q_{1}\right)\right\rangle$ and $y_{1}, y_{2}, \ldots, y_{6}$ be six points of $Q_{2}$ such that $\left\langle\epsilon^{*}\left(y_{1}\right), \epsilon^{*}\left(y_{2}\right), \ldots, \epsilon^{*}\left(y_{6}\right)\right\rangle=\left\langle\epsilon^{*}\left(Q_{2}\right)\right\rangle$. Put $\Omega:=$ $\Omega\left(Q_{1}, Q_{2}\right)$ and let $R_{1}, R_{2}, R_{3}, R_{4}$ be four quads of $\mathcal{P}_{\Omega}$ forming a generating set of points of the geometry $\mathcal{S}_{\Omega}$. For every $i \in\{1,2,3,4\}$, let $z_{i}$ and $z_{i}^{\prime}$ be two points of $R_{i} \backslash \bigcup_{Q \in \Omega} Q$ such that $z_{i}^{\prime}$ is not contained in the subspace of $R_{i}$ generated by $z_{i}, R_{i} \cap Q_{1}$ and $R_{i} \cap Q_{2}$. Then the 20 points $\epsilon^{*}\left(x_{1}\right), \epsilon^{*}\left(x_{2}\right), \ldots, \epsilon^{*}\left(x_{6}\right), \epsilon^{*}\left(y_{1}\right), \epsilon^{*}\left(y_{2}\right), \ldots, \epsilon^{*}\left(y_{6}\right), \epsilon^{*}\left(z_{1}\right), \epsilon^{*}\left(z_{1}^{\prime}\right), \epsilon^{*}\left(z_{2}\right)$, $\epsilon^{*}\left(z_{2}^{\prime}\right), \epsilon^{*}\left(z_{3}\right), \epsilon^{*}\left(z_{3}^{\prime}\right), \epsilon^{*}\left(z_{4}\right), \epsilon^{*}\left(z_{4}^{\prime}\right)$ form a basis of $\Sigma^{*} \cong \mathrm{PG}(19, \mathbb{F})$.

Proof. It suffices to prove that the subspace $\Sigma$ generated by these twenty points coincides with $\Sigma^{*}$. Put $X:=\epsilon^{*-1}\left(\epsilon^{*}(\mathcal{P}) \cap \Sigma\right)$ where $\mathcal{P}$ denotes the point set of $D H\left(5, \mathbb{F}^{\prime}\right)$. Then $X$ is a subspace of $\operatorname{DH}\left(5, \mathbb{F}^{\prime}\right)$ containing $Q_{1}, Q_{2}$ and $\left\{z_{1}, z_{1}^{\prime}, z_{2}, z_{2}^{\prime}, z_{3}, z_{3}^{\prime}, z_{4}, z_{4}^{\prime}\right\}$. If $S_{1}$ and $S_{2}$ are two disjoint quads of $D H\left(5, \mathbb{F}^{\prime}\right)$ and $S \in \Omega\left(S_{1}, S_{2}\right)$, then the points of $S$ are covered by the lines meeting $S_{1}$ and $S_{2}$. This implies the following:

(*) If $S_{1}$ and $S_{2}$ are two disjoint quads contained in $X$, then also every $S \in \Omega\left(S_{1}, S_{2}\right)$ is contained in $X$.

By Property $(*)$, every $Q \in \Omega=\Omega\left(Q_{1}, Q_{2}\right)$ is contained in $X$. Now, for every $i \in$ $\{1,2,3,4\}$, let $G_{i}$ be the set of points of $R_{i}$ contained in a quad of $\Omega$. Then $G_{i}$ is a full subgrid of $\widetilde{R_{i}}$. We denote by $\sigma_{i}$ the $Q(4, \mathbb{F})$-subquadrangle of $\widetilde{R_{i}}$ generated by $\sigma_{i}$ and $z_{i}$. Then $z_{i}^{\prime} \in R_{i} \backslash \sigma_{i}$ and so $R_{i}$ is generated by $\sigma_{i}$ and $z_{i}^{\prime}$. Since $G_{i} \subseteq X$, we have $\sigma_{i} \subseteq X$ and hence $R_{i} \subseteq X$. Since $\left\{R_{1}, R_{2}, R_{3}, R_{4}\right\}$ is a generating set of the point-line geometry $\mathcal{S}_{\Omega}$, every point of $\mathcal{S}_{\Omega}$ is contained in $X$ by Property (*). Lemma 3.6(2) now implies that $X$ coincides with the whole point set of $\operatorname{DH}\left(5, \mathbb{F}^{\prime}\right)$. So, $\Sigma=\Sigma^{*}$.

Lemma 3.9 If $Q_{1}$ and $Q_{2}$ are two disjoint quads of $\Delta$, and $R_{1}, R_{2}, R_{3}$ are three mutually disjoint quads meeting $Q_{1}$ and $Q_{2}$ in lines such that $R_{3}$ is disjoint from any quad of $\Omega\left(R_{1}, R_{2}\right)$, then $\left\langle\epsilon^{*}\left(R_{1}\right), \epsilon^{*}\left(R_{2}\right), \epsilon^{*}\left(R_{3}\right)\right\rangle$ is 17-dimensional.

Proof. Put $L_{i}=R_{i} \cap Q_{1}, i \in\{1,2,3\}$. If $\theta$ is an isomorphism between the point-line dual of $\widetilde{Q_{1}}$ and $H\left(3, \mathbb{F}^{\prime}\right)$, then $L_{1}^{\theta}, L_{2}^{\theta}, L_{3}^{\theta}$ are three points of $H\left(3, \mathbb{F}^{\prime}\right)$ which generate a plane intersecting $H\left(3, \mathbb{F}^{\prime}\right)$ in a unital. By Proposition 3.7, $L_{1}, L_{2}, L_{3}$ are contained in a unique regular spread $S$. Let $\mathcal{A}$ denote the set of all quads $A$ meeting $Q_{1}$ and $Q_{2}$ such that $A \cap Q_{1} \in S$, and let $\mathcal{B}$ be the collection of all sets $\Omega\left(A_{1}, A_{2}\right)$, where $A_{1}$ and $A_{2}$ are two distinct elements of $\mathcal{A}$. Then $(\mathcal{A}, \mathcal{B})$ determines a linear space, isomorphic to a linear space induced on a unital by its hyperbolic lines. If $|\mathbb{F}| \geq 3$, then Proposition 2.8 implies that $\left\{R_{1}, R_{2}, R_{3}\right\}$ generates the point-line geometry determined by $(\mathcal{A}, \mathcal{B})$. In fact, the latter claim is still valid if $|\mathbb{F}|=2$. Indeed, if $|\mathbb{F}|=2$, then the linear space is isomorphic to an affine plane of order 3 , and $\operatorname{AG}(2,3)$ is generated by any three of its points, not on the same line. So, we see that if $Z$ is the union of all quads in $\mathcal{A}$, then 
$\left\langle\epsilon^{*}(Z)\right\rangle=\left\langle\epsilon^{*}\left(R_{1}\right), \epsilon^{*}\left(R_{2}\right), \epsilon^{*}\left(R_{3}\right)\right\rangle$. So, $\left\langle\epsilon^{*}(Z)\right\rangle$ has dimension at most 17 . The point-line geometry $\widetilde{Z}$ induced on $Z$ is a so-called glued near hexagon of type $Q(5, \mathbb{F}) \otimes Q(5, \mathbb{F})$. From [10, Corollary 4.29], it can be deduced that $\left\langle\epsilon^{*}(Z)\right\rangle$ is 17 -dimensional if $\mathbb{F}$ is finite. In fact, by relying on Lemma 3.8 we can see that the latter claim is still valid if $\mathbb{F}$ is infinite. If $|\mathbb{F}| \geq 3$, then by Section 2 (see proof of Proposition 2.13), we know that there exists a line $L_{4}$ in $Q_{1}$ such that $\left\{L_{1}^{\theta}, L_{2}^{\theta}, L_{3}^{\theta}, L_{4}^{\theta}\right\}$ is a generating set of $H\left(3, \mathbb{F}^{\prime}\right)$. We denote by $R_{4}$ the unique quad through $L_{4}$ meeting $Q_{2}$. So, if $\Omega=\Omega\left(Q_{1}, Q_{2}\right)$, then $\left\{R_{1}, R_{2}, R_{3}, R_{4}\right\}$ is a generating set of the point-line geometry $\mathcal{S}_{\Omega}$. By Lemma 3.8, there exist two points $z_{4}, z_{4}^{\prime} \in R_{4}$ such that $\Sigma^{*}=\left\langle\epsilon^{*}\left(R_{1}\right), \epsilon^{*}\left(R_{2}\right), \epsilon^{*}\left(R_{3}\right), \epsilon^{*}\left(z_{4}\right), \epsilon^{*}\left(z_{4}^{\prime}\right)\right\rangle$. This implies that $\left\langle\epsilon^{*}\left(R_{1}\right), \epsilon^{*}\left(R_{2}\right), \epsilon^{*}\left(R_{3}\right)\right\rangle$ has dimension at least 17 , and hence precisely 17 .

Recall that the set of all points $\langle\bar{x}\rangle \in \mathrm{PG}\left(V_{6}^{\prime}\right)$ for which $h_{3}(\bar{x}, \bar{x})=0$ is a nonsingular Hermitian variety $H\left(5, \mathbb{F}^{\prime}\right)$ of $\mathrm{PG}\left(V_{6}^{\prime}\right)$, and that $\Delta=D H\left(5, \mathbb{F}^{\prime}\right)$ is the associated dual polar space.

Suppose $x$ is a point of $\Delta$, i.e. a plane $\alpha$ of $\mathrm{PG}\left(V_{6}^{\prime}\right)$ contained in $H\left(5, \mathbb{F}^{\prime}\right)$. Then there exist natural bijective correspondences between the points of $\alpha$ and the quads of $\Delta$ through $x$ on the one hand, and the lines of $\alpha$ and the lines of $\Delta$ through $x$ on the other hand. So, the lines and quads of $\Delta$ through $x$ define a projective plane $\operatorname{Res}(x) \cong \operatorname{PG}\left(2, \mathbb{F}^{\prime}\right)$. Now, choose vectors $\bar{e}_{1}, \bar{e}_{2}, \bar{e}_{3} \in V_{6}^{\prime}$ such that $\alpha=\left\langle\bar{e}_{1}, \bar{e}_{2}, \bar{e}_{3}\right\rangle$. If $\left(a_{1}, a_{2}, a_{3}\right) \in \mathbb{F}^{\prime 3} \backslash\{(0,0,0)\}$, then the set of all points $\left\langle u_{1} \bar{e}_{1}+u_{2} \bar{e}_{2}+u_{3} \bar{e}_{3}\right\rangle$ of $\alpha$ for which $a_{1} u_{1}+a_{2} u_{2}+a_{3} u_{3}=0$ is a line $L\left(a_{1}, a_{2}, a_{3}\right)$ of $\alpha$.

Suppose now that $a_{i j}$ with $i, j \in\{1,2,3\}$ are elements of $\mathbb{F}^{\prime}$ satisfying $a_{i j}^{\psi}=a_{j i}$ for all $i, j \in\{1,2,3\}$. Then the set of all points $\left\langle u_{1} \bar{e}_{1}+u_{2} \bar{e}_{2}+u_{3} \bar{e}_{3}\right\rangle$ of $\alpha$ satisfying $\sum_{1 \leq i, j \leq 3} a_{i j} u_{i} u_{j}^{\psi}=0$ is a Hermitian curve of $\alpha$ (possibly coinciding with $\alpha$ if $a_{i j}=0$, $\forall i, j \in\{1,2,3\})$, which corresponds to a set of quads through $x$. We denote by $\Upsilon_{q}(x)$ the set of all sets of quads of $\Delta$ through $x$ that can be obtained in this way. The set of all lines $L\left(u_{1}, u_{2}, u_{3}\right)$ of $\alpha$ satisfying $\sum_{1 \leq i, j \leq 3} a_{i j} u_{i} u_{j}^{\psi}=0$ is a Hermitian curve of the dual plane of $\alpha$ (possibly coinciding with the whole set of lines of $\alpha$ if $a_{i j}=0, \forall i, j \in\{1,2,3\}$ ), which corresponds to a set of lines through $x$. We denote by $\Upsilon_{l}(x)$ the set of all sets of lines of $\Delta$ through $x$ that can be obtained in this way.

Lemma 3.10 Let $x$ and $y$ be two opposite points of $\Delta$. Let $\mathcal{Q}$ be a set of quads through $x$ and $\mathcal{L}$ a collection of lines through $y$ such that a quad $Q$ through $x$ belongs to $\mathcal{Q}$ if and only if the unique line through y meeting $Q$ belongs to $\mathcal{L}$. Then $\mathcal{Q} \in \Upsilon_{q}(x)$ if and only if $\mathcal{L} \in \Upsilon_{l}(x)$.

Proof. Let $\alpha$ and $\beta$ be the planes of $\mathrm{PG}\left(V_{6}^{\prime}\right)$ corresponding to $x$ and $y$, respectively. Let $\left(\bar{e}_{1}, \bar{f}_{1}, \bar{e}_{2}, \bar{f}_{2}, \bar{e}_{3}, \bar{f}_{3}\right)$ be a basis of $V_{6}^{\prime}$ such that $\alpha=\left\langle\bar{e}_{1}, \bar{e}_{2}, \bar{e}_{3}\right\rangle, \beta=\left\langle\bar{f}_{1}, \bar{f}_{2}, \bar{f}_{3}\right\rangle, h_{3}\left(\bar{e}_{i}, \bar{e}_{j}\right)=$ $h_{3}\left(\bar{f}_{i}, \bar{f}_{j}\right)=0$ and $h_{3}\left(\bar{e}_{i}, \bar{f}_{j}\right)=\delta_{i j}$ for all $i, j \in\{1,2,3\}$. If $Q$ is a quad through $x$ for which $\left\langle u_{1} \bar{e}_{1}+u_{2} \bar{e}_{2}+u_{3} \bar{e}_{3}\right\rangle$ is the corresponding point of $\alpha$, if $L$ is the unique line through $y$ meeting $Q$ and if $L^{\prime}$ is the unique line of $\beta$ corresponding to $L$, then $L^{\prime}$ consists of all points $\left\langle x_{1} \bar{f}_{1}+x_{2} \bar{f}_{2}+x_{3} \bar{f}_{3}\right\rangle$ for which $u_{1}^{\psi} x_{1}+u_{2}^{\psi} x_{2}+u_{3}^{\psi} x_{3}=0$. The lemma then follows from the fact that a point $\left\langle u_{1} \bar{e}_{1}+u_{2} \bar{e}_{2}+u_{3} \bar{e}_{3}\right\rangle$ of $\alpha$ belongs to a Hermitian curve defined 
by a $\psi$-Hermitian form of $\left\langle\bar{e}_{1}, \bar{e}_{2}, \bar{e}_{3}\right\rangle$ if and only if the point $\left\langle u_{1}^{\psi} \bar{f}_{1}+u_{2}^{\psi} \bar{f}_{2}+u_{3}^{\psi} \bar{f}_{3}\right\rangle$ of $\beta$ belongs to a Hermitian curve defined by a $\psi$-Hermitian form of $\left\langle\bar{f}_{1}, \bar{f}_{2}, \bar{f}_{3}\right\rangle$.

If $H$ is a hyperplane of $\Delta$ and $x \in H$, then $\Lambda_{H}(x)$ denotes the set of lines through $x$ contained in $H$. The following was proved in [2, Section 3.4].

Proposition 3.11 Suppose $H$ is a hyperplane of $\Delta$ arising from its Grassmann embedding and let $x \in H$. Then $\Lambda_{H}(x) \in \Upsilon_{l}(x)$.

The following proposition can easily be deduced from Lemma 3.10 and Proposition 3.11.

Proposition 3.12 Suppose $H$ is a hyperplane of $\Delta$ arising from the Grassmann embedding containing a deep point $x$. Then the set $\mathcal{D}$ of deep quads through $x$ belongs to $\Upsilon_{q}(x)$.

Proof. Suppose first that $\Gamma_{3}(x) \cap H=\emptyset$. Then $H$ is contained in the singular hyperplane $H_{x}$ with deepest point $x$, and hence coincides with $H_{x}$ since hyperplanes of thick dual polar spaces are maximal proper subspaces. It follows that $\mathcal{D}$ consists of all quads through $x$. Hence, $\mathcal{D} \in \Upsilon_{q}(x)$.

Suppose $y \in \Gamma_{3}(x) \cap H$. Then $\Lambda_{H}(y) \in \Upsilon_{l}(y)$. Since every quad through $x$ is deep or singular, a line $L$ through $y$ belongs to $\Lambda_{H}(y)$ if and only if the unique quad through $x$ meeting $L$ belongs to $\mathcal{D}$. The corollary then follows from Lemma 3.10.

If $x$ and $y$ are two opposite points of $\Delta$, then the subspaces $\Sigma_{x}:=\left\langle\epsilon^{*}\left(x^{\perp}\right)\right\rangle$ and $\Sigma_{y}:=$ $\left\langle\epsilon^{*}\left(y^{\perp}\right)\right\rangle$ are two disjoint 9-dimensional subspaces of $\Sigma^{*}$. Let $\delta$ be an arbitrary element of $\mathbb{F}^{\prime} \backslash \mathbb{F}$.

Proposition 3.13 Let $x$ and $y$ be two opposite points of $\Delta$, and let $\mathcal{L}$ be a set of lines through $y$ defining a nonempty nonsingular Hermitian curve of $\operatorname{Res}(y)$ belonging to $\Upsilon_{l}(y)$. Then $\left\langle\epsilon^{*}\left(\bigcup_{L \in \mathcal{L}} L\right)\right\rangle$ is a hyperplane of $\Sigma_{y}$ and there exists a unique hyperplane $H$ of $\Delta$ arising from $\epsilon^{*}$ such that $x$ is a deep point, $y \in H$ and $\Lambda_{H}(y)=\mathcal{L}$.

Proof. Let $V^{*}$ be a 20 -dimensional vector space over $\mathbb{F}$ such that $\Sigma^{*}=\operatorname{PG}\left(V^{*}\right)$. Let $V_{x}$ and $V_{y}$ be the 10-dimensional subspaces of $V^{*}$ such that $\Sigma_{x}=\operatorname{PG}\left(V_{x}\right)$ and $\Sigma_{y}=\operatorname{PG}\left(V_{y}\right)$. Then $V^{*}=V_{x} \oplus V_{y}$ by [2, Corollary 3.3].

Let $\alpha$ be the plane of $H\left(5, \mathbb{F}^{\prime}\right)$ corresponding to $y$ and let $U$ be the 3-space of $V_{6}^{\prime}$ corresponding to $\alpha$. We choose an ordered basis $B=\left(\bar{f}_{1}, \bar{f}_{2}, \bar{f}_{3}\right)$ in $U$, and we shall denote by $\left(X_{1}, X_{2}, X_{3}\right)$ the coordinates of a vector of $U$ with respect to this basis. We also choose an ordered basis $B^{\prime}=\left(\bar{g}_{1}, \bar{g}_{2}, \ldots, \bar{g}_{10}\right)$ in $V_{y}$ such that $\left\langle\bar{g}_{10}\right\rangle=\epsilon^{*}(y)$, and we shall denote by $\left(Y_{1}, Y_{2}, \ldots, Y_{10}\right)$ the coordinates of a vector of $V_{y}$ with respect to this basis.

If $W$ is a subspace of co-dimension at most 1 of $V_{y}$ containing $\epsilon^{*}(y)$, then we denote by $\mathcal{L}_{W}$ the set of lines of $\Delta$ through $y$ that are mapped by $\epsilon^{*}$ into $\operatorname{PG}(W)$. Then $\mathcal{L}_{W} \in \Upsilon_{l}(y)$ by Proposition 3.11. From [2, Section 3.4], we know that the bases $B$ and $B^{\prime}$ can be chosen in such a way that if $W$ has equation $a_{11} Y_{1}+a_{22} Y_{2}+a_{33} Y_{3}+\left(a_{12}+a_{12}^{\psi}\right) Y_{4}+\left(\delta a_{12}+\right.$ $\left.\delta^{\psi} a_{12}^{\psi}\right) Y_{5}+\left(a_{13}+a_{13}^{\psi}\right) Y_{6}+\left(\delta a_{13}+\delta^{\psi} a_{13}^{\psi}\right) Y_{7}+\left(a_{23}+a_{23}^{\psi}\right) Y_{8}+\left(\delta a_{23}+\delta^{\psi} a_{23}^{\psi}\right) Y_{9}=0$ for some 
$a_{11}, a_{22}, a_{33} \in \mathbb{F}$ and some $a_{12}, a_{13}, a_{23} \in \mathbb{F}^{\prime}$, then $\mathcal{L}_{W}$ consists of all lines described by equations $u_{1} X_{1}+u_{2} X_{2}+u_{3} X_{3}=0$ for which the coefficients $u_{1}, u_{2}, u_{3}$ satisfy the equation $\sum_{1 \leq i, j \leq 3} a_{i j} u_{i} u_{j}^{\psi}=0$, where $a_{21}=a_{12}^{\psi}, a_{31}=a_{13}^{\psi}$ and $a_{32}=a_{23}^{\psi}$.

Now, if $\sum_{1 \leq i, j \leq 3} a_{i j} u_{i} u_{j}^{\psi}=0$, with $a_{j i}=a_{i j}^{\psi}$ for all $i, j \in\{1,2,3\}$, describes a nonsingular nonempty Hermitian curve, then the coefficients $a_{i j}$ are uniquely determined by the Hermitian curve, up to a factor in $\mathbb{F} \backslash\{0\}$. So, there must exist a unique hyperplane $W^{*}$ in $V_{y}$ containing $\epsilon^{*}(y)$ such that $\mathcal{L}_{W^{*}}=\mathcal{L}$. If $U$ is the hyperplane of $\Sigma^{*}$ generated by $\Sigma_{x}$ and $\operatorname{PG}\left(W^{*}\right)$, then we see that $H_{U}$ is a hyperplane of $\Delta$ such that $x$ is a deep point and $\Lambda_{H_{U}}(y)=\mathcal{L}$. Moreover, $H_{U}$ has to be the unique hyperplane of $\Delta$ arising from $\epsilon^{*}$ having these properties.

We still need to show that $\left\langle\epsilon^{*}\left(\bigcup_{L \in \mathcal{L}} L\right)\right\rangle$ is a hyperplane of $\Sigma_{y}$. Suppose that this is not the case, then besides $\mathrm{PG}\left(W^{*}\right)$ there exists another hyperplane $\mathrm{PG}\left(W_{1}\right)$ containing this subspace. But then $\mathcal{L}=\mathcal{L}_{W^{*}}$ would be properly contained in $\mathcal{L}_{W_{1}}$, which is impossible since both these sets are Hermitian curves of $\operatorname{Res}(y)$ with the former one being nonsingular and nonempty and the latter one being distinct from the whole point set of $\operatorname{Res}(y)$.

\section{Proofs of Theorems 1.1 and 1.2}

\subsection{Proof of Theorem 1.1 in the case not every quad of $\Omega$ is deep}

Suppose $|\mathbb{F}| \geq 3$. Let $\Omega$ be a hyperbolic set of quads of $\Delta=D H\left(5, \mathbb{F}^{\prime}\right)$ and let $\mathcal{Q}$ be the set of all quads of $\Delta$ which either belong to $\Omega$ or intersect each quad of $\Omega$ in a line. For every $Q \in \mathcal{Q}$, let $\mathcal{C}_{Q}$ be a set of classical hyperplanes of $\widetilde{Q}$ such that the conditions (1) and (2) of Theorem 1.1 are satisfied. Let $\mathcal{C}$ denote the set of all hyperplanes $H$ of $\Delta$ such that $H \cap Q \in \mathcal{C}_{Q}$ for every quad $Q \in \mathcal{Q}$ not contained in $H$.

Let $Z$ denote the union of all quads $Q$ of $\Omega$, and let $\widetilde{Z}$ denote the point-line geometry induced on $Z$ (by those lines of $\Delta$ that are completely contained in $Z$ ). Let $\epsilon_{Z}$ denote the embedding of $\widetilde{Z}$ induced by the Grassmann embedding $\epsilon^{*}: \Delta \rightarrow \Sigma^{*}$ of $\Delta=D H\left(5, \mathbb{F}^{\prime}\right)$.

We will first prove Theorem 1.1 in the case the hyperplane $H \in \mathcal{C}$ does not contain $Z$. Let $Q_{1}$ and $Q_{2}$ be two disjoint quads of $\Omega$ such that:

If $H$ contains a (necessarily unique) member of $\Omega$, then this element of $\Omega$ is equal to $Q_{1}$.

Put $\Sigma_{1}=\left\langle\epsilon^{*}\left(Q_{1}\right)\right\rangle$ and $\Sigma_{2}=\left\langle\epsilon^{*}\left(Q_{2}\right)\right\rangle$. Then $\left\langle\Sigma_{1}, \Sigma_{2}\right\rangle$ is the co-domain of $\epsilon_{Z}$. Now, put $\sigma_{i}=H \cap Q_{i}, i \in\{1,2\}$. Then $\sigma_{2} \neq Q_{2}$. If $\sigma_{i} \neq Q_{i}$, then $\sigma_{i}$ is a classical hyperplane of $\widetilde{Q_{i}}$ and hence $\left\langle\epsilon^{*}\left(\sigma_{i}\right)\right\rangle$ is a hyperplane of $\Sigma_{i}$. For every point $x$ of $Q_{1}$, let $L_{x}$ denote the unique line through $x$ meeting $Q_{2}$. Let $H_{Z}^{*}$ denote the hyperplane $H \cap Z$ of $\widetilde{Z}$.

Lemma 4.1 The hyperplane $H_{Z}^{*}$ arises from the embedding $\epsilon_{Z}$.

Proof. We distinguish two cases.

- Suppose first that $Q_{1} \subseteq H$. Then $H_{Z}^{*}=Q_{1} \cup\left(\bigcup_{Q \in \Omega \backslash\left\{Q_{1}\right\}} \pi_{Q}\left(\sigma_{2}\right)\right)$. On the other hand, let $U$ denote the hyperplane of $\left\langle\Sigma_{1}, \Sigma_{2}\right\rangle$ generated by $\Sigma_{1}$ and $\left\langle\epsilon^{*}\left(\sigma_{2}\right)\right\rangle$, and put 
$H_{Z}=\epsilon^{*-1}\left(\epsilon^{*}(Z) \cap U\right)$. Then $Q_{1} \subseteq H_{Z}$ and $H_{Z} \cap Q_{2}=\sigma_{2}$. Hence, also $H_{Z}=Q_{1} \cup$ $\left(\bigcup_{Q \in \Omega \backslash\left\{Q_{1}\right\}} \pi_{Q}\left(\sigma_{2}\right)\right)$. It follows that $H_{Z}=H_{Z}^{*}$. Hence, $H_{Z}^{*}$ arises from the embedding

- Next, suppose that $Q_{1} \nsubseteq H$. We show that $\sigma_{1} \neq \pi_{Q_{1}}\left(\sigma_{2}\right)$. Suppose $\sigma_{1}=\pi_{Q_{1}}\left(\sigma_{2}\right)$. Let $u$ be a point of $Q_{1} \backslash \sigma_{1}, v$ the unique point of $L_{u}$ contained in $H$ and $Q^{\prime}$ the unique element of $\Omega$ containing $v$. Then the hyperplane $Q^{\prime} \cap H$ of $\widetilde{Q^{\prime}}$ would contain $\{v\} \cup \pi_{Q^{\prime}}\left(\sigma_{1}\right)$, which is impossible since the hyperplane $\pi_{Q^{\prime}}\left(\sigma_{1}\right)$ of $\widetilde{Q^{\prime}}$ is a maximal proper subspace.

So, $\sigma_{1}$ and $\pi_{Q_{1}}\left(\sigma_{2}\right)$ are two distinct hyperplanes of $\widetilde{Q_{1}}$ belonging to $\mathcal{C}_{Q_{1}}$. Let $\Pi$ denote the unique pencil of classical hyperplanes of $\widetilde{Q}_{1}$ containing $\sigma_{1}$ and $\pi_{Q_{1}}\left(\sigma_{2}\right)$. By Lemma $3.6(3)$, we must have that

$$
\Pi=\left\{\pi_{Q_{1}}(H \cap Q) \mid Q \in \Omega\right\} .
$$

Indeed, since $H \cap Q \in \mathcal{C}_{Q}$ is a classical hyperplane of $\widetilde{Q}$, also $\pi_{Q_{1}}(H \cap Q)$ is a classical hyperplane of $\widetilde{Q}_{1}$. Moreover, the set $\left\{\pi_{Q_{1}}(H \cap Q) \mid Q \in \Omega\right\}$ contains $\sigma_{1}$ and $\pi_{Q_{1}}\left(\sigma_{2}\right)$. Now, $\sigma_{1}$ and $\pi_{Q_{1}}\left(\sigma_{2}\right)$ are two distinct hyperplanes of $\widetilde{Q_{1}}$, each of which is a singular hyperplane, an ovoid or a $Q(4, \mathbb{K})$-subquadrangle. Considering all mutual positions of $\sigma_{1}$ and $\pi_{Q_{1}}\left(\sigma_{2}\right)$ immediately reveals that there must exist a line $L$ in $Q_{1}$ that intersects $\sigma_{1}$ and $\pi_{Q_{1}}\left(\sigma_{2}\right)$ in two distinct singletons. Let $x$ be a point of $L$ not contained in $\sigma_{1} \cup \pi_{Q_{1}}\left(\sigma_{2}\right)$, and let $y$ denote the unique point of $H$ contained on the line $L_{x}$. Let $U$ denote the hyperplane of $\left\langle\Sigma_{1}, \Sigma_{2}\right\rangle$ generated by $\epsilon^{*}\left(\sigma_{1}\right), \epsilon^{*}\left(\sigma_{2}\right)$ and $e(y)$. Put $H_{Z}:=\epsilon^{*-1}\left(\epsilon^{*}(Z) \cap U\right)$. By Lemma $3.6(3)$, we again have

$$
\Pi=\left\{\pi_{Q_{1}}\left(H_{Z} \cap Q\right) \mid Q \in \Omega\right\} .
$$

(Indeed, since $H_{Z} \cap Q$ is a classical hyperplane of $\widetilde{Q}$, also $\pi_{Q_{1}}\left(H_{Z} \cap Q\right)$ is a classical hyperplane of $\widetilde{Q}_{1}$. Moreover, the set $\left\{\pi_{Q_{1}}\left(H_{Z} \cap Q\right) \mid Q \in \Omega\right\}$ contains $\sigma_{1}$ and $\pi_{Q_{1}}\left(\sigma_{2}\right)$.) Now, let $Q_{L}$ denote the unique quad through $L$ meeting $Q_{2}$ in a line. We denote by $G_{L}$ the full subgrid $Q_{L} \cap Z$. Let $u$ denote the unique point in $L \cap H$, let $v$ denote the unique point in $\pi_{Q_{2}}(L) \cap H$. Then $u, v$ and $y$ are contained in $H_{Z} \cap H_{Z}^{*}$. Since both $H_{Z} \cap Q_{L}$ and $H_{Z}^{*} \cap Q_{L}$ are classical hyperplanes of $\widetilde{Q_{L}}$, and $G_{L}$ is neither contained in $H_{Z}$, nor in $H_{Z}^{*}$, we have

$$
H_{Z} \cap G_{L}=H_{Z}^{*} \cap G_{L}
$$

Note that $H_{Z} \cap G_{L}=H_{Z}^{*} \cap G_{L}$ is an ovoid of the point-line geometry $\widetilde{G_{L}}$ induced on $G_{L}$, i.e., every line of $\widetilde{G_{L}}$ intersects $H_{Z} \cap G_{L}=H_{Z}^{*} \cap G_{L}$ in a singleton. The conditions (2), (3) and (4) now imply that $H_{Z}=H_{Z}^{*}$. Indeed, for this to be valid, we need to show that $H_{Z} \cap Q_{3}=H_{Z}^{*} \cap Q_{3}$ for every $Q_{3} \in \Omega$. If $w$ denotes the unique point of the ovoid $H_{Z} \cap G_{L}=H_{Z}^{*} \cap G_{L}$ of $\widetilde{G_{L}}$ contained in $Q_{3} \cap Q_{L}$, and if $\sigma$ denotes the unique member of $\Pi$ containing $\pi_{Q_{1}}(w)$, then (2) and (3) imply that $H_{Z} \cap Q_{3}=\pi_{Q_{3}}(\sigma)=H_{Z}^{*} \cap Q_{3}$. Since we now know that $H_{Z}=H_{Z}^{*}$, the hyperplane $H_{Z}^{*}$ must arise from the embedding $\epsilon_{Z}$.

Let $\mathcal{P}_{\Omega}$ and $\mathcal{L}_{\Omega}$ be as defined in Section 3.3. For every proper subset $X$ of $Q_{2}$, let $\mathcal{A}_{X}$ denote the point-line geometry whose points are those quads $Q \in \mathcal{P}_{\Omega}$ for which the line $Q \cap Q_{2}$ is not contained in $X$ and whose lines are those elements $\Omega^{\prime} \in \mathcal{L}_{\Omega}$ 
with the property that $Q \cap Q_{2}$ is not contained in $X$ for every $Q \in \Omega^{\prime}$. The incidence relation is containment. By Proposition 3.4 and invoking the isomorphism described in Lemma 3.6(1), we know that there exists a subset $\left\{R_{1}, R_{2}, R_{3}, R_{4}\right\}$ of size 4 of $\mathcal{P}_{\Omega}$ generating the geometry $\mathcal{A}_{\sigma_{2}}$. For every $i \in\{1,2,3,4\}$, let $G_{i}$ be the full subgrid $R_{i} \cap Z$. Then $H \cap G_{i}$ is a certain hyperplane of $\widetilde{G}_{i}$. For every $i \in\{1,2,3,4\}, H \cap R_{i}$ is a classical hyperplane of $\widetilde{R_{i}}$ and hence there exist two points $y_{i}$ and $z_{i}$ of $\left(H \cap R_{i}\right) \backslash G_{i}$ such that $\left\langle\epsilon^{*}\left(H \cap R_{i}\right)\right\rangle=\left\langle\epsilon^{*}\left(H \cap G_{i}\right), \epsilon^{*}\left(y_{i}\right), \epsilon^{*}\left(z_{i}\right)\right\rangle$. By Lemma 3.8, we know that $W=$ $\left\langle\epsilon^{*}\left(H_{Z}^{*}\right), \epsilon^{*}\left(y_{1}\right), \epsilon^{*}\left(z_{1}\right), \epsilon^{*}\left(y_{2}\right), \epsilon^{*}\left(z_{2}\right), \epsilon^{*}\left(y_{3}\right), \epsilon^{*}\left(z_{3}\right), \epsilon^{*}\left(y_{4}\right), \epsilon^{*}\left(z_{4}\right)\right\rangle$ is a hyperplane of $\Sigma^{*}$. Let $H^{*}$ denote the set of all points of $D H\left(5, \mathbb{F}^{\prime}\right)$ that are mapped by $\epsilon^{*}$ into $W$. Then $H^{*}$ is a hyperplane arising from the Grassmann embedding. Our aim will be to show that $H=H^{*}$. Since $\left\langle\epsilon^{*}(Z), \epsilon^{*}\left(y_{1}\right), \epsilon^{*}\left(z_{1}\right), \epsilon^{*}\left(y_{2}\right), \epsilon^{*}\left(z_{2}\right), \epsilon^{*}\left(y_{3}\right), \epsilon^{*}\left(z_{3}\right), \epsilon^{*}\left(y_{4}\right), \epsilon^{*}\left(z_{4}\right)\right\rangle=\Sigma^{*}$, we have that $H^{*} \cap Z=H \cap Z$. We call a quad $R \in \mathcal{P}_{\Omega}$ good if $R \cap H=R \cap H^{*}$. By Lemma 3.6(2), in order to show that $H=H^{*}$, it suffices to prove that all quads of $\mathcal{P}_{\Omega}$ are good. By construction of the hyperplane $H^{*}$, the quads $R_{1}, R_{2}, R_{3}$ and $R_{4}$ are good.

Lemma 4.2 If $\Omega^{\prime} \in \mathcal{L}_{\Omega}$ is a line of $\mathcal{A}_{\sigma_{2}}$ containing two good quads, then all quads of $\Omega^{\prime}$ are good.

Proof. Let $G$ denote the full subgrid of $Q_{2}$ containing all lines $Q_{2} \cap Q, Q \in \Omega^{\prime}$. Suppose $S_{1}$ and $S_{2}$ are two distinct good quads of $\Omega^{\prime}$. Since $\Omega^{\prime}$ is a line of $\mathcal{A}_{\sigma_{2}}$, the intersection $G \cap H$ is an ovoid $O$ of $\widetilde{G}$. So, $S_{1} \cap H$ and $\pi_{S_{1}}\left(S_{2} \cap H\right)$ are two distinct hyperplanes of $\mathcal{C}_{S_{1}}$. Let $\Pi$ denote the unique pencil of classical hyperplanes of $\widetilde{S}_{1}$ containing $S_{1} \cap H$ and $\pi_{S_{1}}\left(S_{2} \cap H\right)$. By Lemma 3.6(3), $\Pi=\left\{\pi_{S_{1}}(S \cap H) \mid S \in \Omega^{\prime}\right\}^{2}$. On the other hand, since $S_{2} \cap H^{*}=S_{2} \cap H$ and $S_{1} \cap H^{*}=S_{1} \cap H$, also $\left\{\pi_{S_{1}}\left(S \cap H^{*}\right) \mid S \in \Omega^{\prime}\right\}$ is a pencil of classical hyperplanes of $\widetilde{S}_{1}$ containing $S_{1} \cap H$ and $\pi_{S_{1}}\left(S_{2} \cap H\right)$. It follows that

$$
\left\{\pi_{S_{1}}(S \cap H) \mid S \in \Omega^{\prime}\right\}=\left\{\pi_{S_{1}}\left(S \cap H^{*}\right) \mid S \in \Omega^{\prime}\right\} .
$$

Now, let $S_{3}$ be an arbitrary element of $\Omega^{\prime} \backslash\left\{S_{1}, S_{2}\right\}$, and let $u$ denote the unique element of $O \cap\left(S_{3} \cap Q_{2}\right)$. Since $u \in H \cap H^{*}$, we would have

$$
S_{3} \cap H=\pi_{S_{3}}(\sigma)=S_{3} \cap H^{*},
$$

where $\sigma$ is the unique element of $\Pi$ containing $\pi_{S_{1}}(u)$. Hence, every quad of $\Omega^{\prime}$ is good.

For every line $L$ contained in $Q_{2}$, let $Q_{L}$ denote the unique quad through $L$ meeting $Q_{1}$. Since $\left\{R_{1}, R_{2}, R_{3}, R_{4}\right\}$ is a generating set of the geometry $\mathcal{A}_{\sigma_{2}}$, and the quads $R_{1}, R_{2}, R_{3}$, $R_{4}$ are good, Lemma 4.2 implies the following:

If $L$ is a line of $Q_{2}$ not contained in $\sigma_{2}$, then the quad $Q_{L}$ is good.

In order to show that all quads of $\mathcal{P}_{\Omega}$ are good, it remains to show that every quad $Q_{L}$ is good, where $L$ is a line of $Q_{2}$ contained in $\sigma_{2}$. Let $G$ be a full subgrid of $Q_{2}$ containing $L$,

\footnotetext{
${ }^{2}$ Indeed, since $S \cap H \in \mathcal{C}_{S}$ is a classical hyperplane of $\widetilde{S}, \pi_{S_{1}}(S \cap H)$ is a classical hyperplane of $\widetilde{S_{1}}$. Moreover, the set $\left\{\pi_{S_{1}}(S \cap H) \mid S \in \Omega^{\prime}\right\}$ contains $S_{1} \cap H$ and $\pi_{S_{1}}\left(S_{2} \cap H\right)$.
} 
but not contained in $\sigma_{2}$, and let $\mathcal{L}$ denote the set of lines of $\widetilde{G}$ parallel with or equal to $L$. Put $\Omega^{\prime}=\left\{Q_{L^{\prime}} \mid L^{\prime} \in \mathcal{L}\right\}$. Then $\Omega^{\prime}$ is a hyperbolic set of quads. Every line of $\mathcal{L} \backslash\{L\}$ is not contained in $\sigma_{2}$, and hence all quads of $\Omega^{\prime} \backslash\left\{Q_{L}\right\}$ are good. By considering all lines meeting all quads of $\Omega^{\prime}$ and using the fact that each such line has either one or all of its points $^{3}$ in $H$, we see that the fact that $H \cap Q=H^{*} \cap Q, \forall Q \in \Omega^{\prime} \backslash\left\{Q_{L}\right\}$, implies that $H \cap Q_{L}=H^{*} \cap Q_{L}$ as well.

\subsection{Proof of Theorem 1.2 and proof of Theorem 1.1 in case all quads of $\Omega$ are deep}

Proposition 4.3 Suppose $|\mathbb{F}| \geq 3$. Let $Q_{1}$ and $Q_{2}$ be two disjoint quads of $D H\left(5, \mathbb{F}^{\prime}\right)$ and suppose $H$ is a hyperplane of $D H\left(5, \mathbb{F}^{\prime}\right)$ such that $Q_{1} \subseteq H$ and $Q_{2} \cap H$ is a classical hyperplane of $\widetilde{Q_{2}}$. Then $H$ arises from the Grassmann embedding of $D H\left(5, \mathbb{F}^{\prime}\right)$.

Proof. Put $\Omega:=\Omega\left(Q_{1}, Q_{2}\right)$ and let $\mathcal{R}$ denote the set of all quads of $D H\left(5, \mathbb{F}^{\prime}\right)$ meeting every quad of $\Omega$ in a line. For every quad $Q \in \Omega$, put $\mathcal{C}_{Q}:=\left\{\pi_{Q}\left(Q_{2} \cap H\right)\right\}$. For every quad $R \in \mathcal{R}$, let $\mathcal{C}_{R}$ denote the set of all (classical) hyperplanes $\sigma$ of $\widetilde{R}$ such that $R \cap Q_{1} \subseteq \sigma$. Then $\mathcal{Q}=\Omega \cup \mathcal{R}$ satisfies the conditions of Theorem 1.1 by Lemma 3.5. Since not every quad of $\Omega$ is contained in $H$ and $H \cap Q \in \mathcal{C}_{Q}$ for every quad $Q \in \mathcal{Q}$ not contained in $H$, the hyperplane $H$ must arise from the Grassmann embedding by Section 4.1.

Proposition 4.4 The extension $H$ of a classical ovoid $O$ of a quad $Q$ of $D H\left(5, \mathbb{F}^{\prime}\right)$ arises from the Grassmann embedding of $\mathrm{DH}\left(5, \mathbb{F}^{\prime}\right)$.

Proof. Since $Q(5, q)$ does not have ovoids for every prime power $q$, the field $\mathbb{F}$ should be infinite. Take a quad $Q^{\prime}$ disjoint from $Q$. Since the map $Q \rightarrow Q^{\prime} ; x \mapsto \pi_{Q^{\prime}}(x)$ defines an isomorphism between $\widetilde{Q}$ and $\widetilde{Q^{\prime}}$, the set $Q^{\prime} \cap H=\pi_{Q^{\prime}}(O)$ necessarily is a classical ovoid of $\widetilde{Q^{\prime}}$. Proposition 4.3 now implies that $H$ arises from the Grassmann embedding of $\operatorname{DH}\left(5, \mathbb{F}^{\prime}\right)$.

Since $Q(4, \mathbb{F})$-subquadrangles and singular hyperplanes of $Q(4, \mathbb{F})$ are classical hyperplanes, Propositions 3.1, 4.3 and 4.4 imply Theorem 1.2:

Corollary 4.5 Suppose $|\mathbb{F}| \geq 3$ and $H$ is a hyperplane of $D H\left(5, \mathbb{F}^{\prime}\right)$ containing a quad $Q$. Then $H$ either arises from the Grassmann embedding of $D H\left(5, \mathbb{F}^{\prime}\right)$ or is the extension of a non-classical ovoid of $\widetilde{Q}$.

Observe that the extension of a non-classical ovoid of a quad of $D H\left(5, \mathbb{F}^{\prime}\right)$ cannot arise from a projective embedding.

Corollary 4.5 has the following consequence, which shows the validity of Theorem 1.1 in the special case all quads of $\Omega$ are deep.

\footnotetext{
${ }^{3}$ If you know for all but one of the points of a line $L$ whether they belong to $H$ or not, then you also know that for the remaining point of $L$.
} 
Corollary 4.6 Suppose $|\mathbb{F}| \geq 3$. If $H$ is a hyperplane of $D H\left(5, \mathbb{F}^{\prime}\right)$ containing two disjoint quads, then $H$ arises from the Grassmann embedding of $D H\left(5, \mathbb{F}^{\prime}\right)$.

\section{Proof of Theorem 1.3}

In this section, we suppose that $H$ is a hyperplane of $\Delta=D H\left(5, \mathbb{F}^{\prime}\right)$ arising from the Grassmann embedding and containing a quad $Q$.

Suppose first that there is some deep point $x$. Then every quad through $x$ is either deep or singular. If $\Gamma_{3}(x) \cap H=\emptyset$, then by Proposition 3.2(1), $H$ must be the singular hyperplane with deepest point $x$. Suppose therefore that there exists a point $y \in \Gamma_{3}(x) \cap H$. Then the deep quads through $x$ are precisely the quads through $x$ meeting a line of $\Lambda_{H}(y)$. If $\Lambda_{H}(y)=\emptyset$, then by Proposition 3.2(2), $H$ must be a semi-singular hyperplane, in contradiction with the existence of deep quads. Therefore, $\Lambda_{H}(y)$ must be a nonempty Hermitian curve of $\operatorname{Res}(y)$ by Proposition 3.11. If this Hermitian curve is singular, then Proposition 3.2(3) implies that $H$ is either a singular hyperplane, the extension of a $Q(4, \mathbb{F})$-subquadrangle of a quad, or the extension of a (necessarily classical) ovoid of a quad. If the Hermitian curve is nonsingular, then $H$ must be a hyperplane as described in Proposition 3.13. So, we know what the hyperplane $H$ is in case there exists a deep point. In the rest of this section, we will therefore make the following assumption:

\section{Assumption: There are no deep points.}

By Proposition 3.11, we know that for every point $x \in H$, the set $\Lambda_{H}(x)$ is a possibly degenerate Hermitian curve of $\operatorname{Res}(x)$. Since $Q \subseteq H$, this implies that for every point $x \in Q, \Lambda_{H}(x)$ is either a line or a Baer pencil of $\operatorname{Res}(x)$. If $\Lambda_{H}(x)$ is a Baer pencil of $\operatorname{Res}(x)$, then there exists a unique line $L$ through $x$ (corresponding with the center of the Baer pencil) having the property that every quad through $L$ is deep or singular with respect to $H$. Every quad through $x$ not containing $L$ is subquadrangular with respect to $H$.

Lemma 5.1 For every point $x$ of $Q, \Lambda_{H}(x)$ is a Baer pencil of $\operatorname{Res}(x)$.

Proof. Suppose that this is not the case. Then there exists a point $x^{*} \in Q$ such that $\Lambda_{H}\left(x^{*}\right)$ is a line of $\operatorname{Res}\left(x^{*}\right)$. So, the lines through $x^{*}$ contained in $H$ are precisely the lines through $x^{*}$ contained in $Q$.

We show that for every $y \in \Gamma_{1}\left(x^{*}\right) \cap Q, \Lambda_{H}(y)$ is a Baer pencil of $\operatorname{Res}(y)$ with center $x^{*} y$. Let $R$ be a quad through $x^{*} y$ distinct from $Q$. Since $\left(x^{*}\right)^{\perp} \cap H \cap R=R \cap Q=x^{*} y$, the quad $R$ is singular and its deepest point $z$ belongs to $x^{*} y \backslash\left\{x^{*}\right\}$. Since $\Lambda_{H}(z)$ cannot be a line of $\operatorname{Res}(z)$, it must be a Baer pencil with center $x^{*} y=x^{*} z$ (since every quad through $x^{*} y$ distinct from $Q$ is singular). Let $L_{1}$ be a line of $Q$ through $x^{*}$ distinct from $x^{*} y$ and let $L_{2}$ be a line of $Q$ through $z$ distinct from $x^{*} y$. Then $L_{1}$ and $L_{2}$ are disjoint. Let $S_{i}, i \in\{1,2\}$, be a quad through $L_{i}$ distinct from $Q$ and let $S_{3}$ be the unique element of $\Omega\left(S_{1}, S_{2}\right)$ containing the point $y$. The quad $S_{1}$ is singular with respect to $H$ since $S_{1} \cap H \cap\left(x^{*}\right)^{\perp}=S_{1} \cap Q$. The quad $S_{2}$ on the other hand is subquadrangular with respect 
to $H$ as it does not contain the line $x^{*} y$. Now, $\Pi=\left\{\pi_{S_{3}}(S \cap H) \mid S \in \Omega\left(S_{1}, S_{2}\right)\right\}$ is a pencil of hyperplanes of $\widetilde{S}_{3}$, each of which contains the line $S_{3} \cap Q$. Since the pencil contains a singular hyperplane (namely $\pi_{S_{3}}\left(S_{1} \cap H\right)$ ) and a subquadrangular hyperplane (namely $\pi_{S_{3}}\left(S_{2} \cap H\right)$ ), precisely one hyperplane of $\Pi$ must be singular by Lemma 3.5. This shows that the hyperplane $S_{3} \cap H$ of $\widetilde{S}_{3}$ is subquadrangular. This implies that $\Lambda_{H}(y)$ is a Baer pencil of $\operatorname{Res}(y)$. The center of the Baer pencil must be $x^{*} y$, since every quad through $x^{*} y$ distinct from $Q$ is singular.

We show that every quad $R$ intersecting $Q$ in a line $L$ not containing $x^{*}$ is subquadrangular. Let $y$ denote the unique point of $L$ collinear with $x^{*}$. Then $\Lambda_{H}(y)$ is a Baer pencil of $\operatorname{Res}(y)$ with center $x^{*} y$. The quads through $x^{*} y$ corresponding to the lines of this Baer pencil each intersect $R$ in a line which is contained in $H$. Every other quad through $x^{*} y$ intersects $R$ in a line which is not contained in $H$. So, $R$ must be a subquadrangular quad.

Now, let $y$ be a point of $Q$ not collinear with $x^{*}$. Then every quad through $y$ distinct from $Q$ is subquadrangular. But that is not possible, since $\Lambda_{H}(y)$ is either a line or a Baer pencil of $\operatorname{Res}(y)$.

Now, let $S$ denote the set of all lines $L \subseteq Q$ having the property that every quad through $L$ is either deep or singular. By Lemma 5.1, every point of $Q$ is contained in a unique element of $S$, i.e. $S$ is a spread of $\widetilde{Q}$.

Lemma 5.2 Every line $L \in S$ is contained in a unique deep quad distinct from $Q$.

Proof. Let $x_{1}$ and $x_{2}$ be two distinct points of $L$. For every $i \in\{1,2\}$, let $L_{i}$ be a line of $Q$ through $x_{i}$ distinct from $L$ and let $R_{i}$ be a quad such that $R_{i} \cap Q=L_{i}$. Then $R_{1}$ and $R_{2}$ are disjoint and every quad of $\Omega\left(R_{1}, R_{2}\right)$ is subquadrangular, since none of these quads contains the line $L \in S$. This implies by Lemma 3.5 and 3.6(3) that $\left(H \cap R_{1}\right) \cap \pi_{R_{1}}\left(H \cap R_{2}\right)$ is a full subgrid. Let $M$ denote the line of this full subgrid that contains $x_{1}$ but is distinct from $L_{1}$. Then $\langle L, M\rangle$ is the unique quad through $L$ distinct from $Q$ that intersects each quad of $\Omega\left(R_{1}, R_{2}\right)$ in a line that is contained in $H$. Since every quad through $L$ is either singular or deep, $\langle L, M\rangle$ must be a deep quad. In fact, one can even say more. The quad $\langle L, M\rangle$ must be the unique deep quad through $L$ distinct from $Q$.

Lemma 5.3 Let $Q^{\prime}$ be a deep quad disjoint from $Q$ and let $L \in S$. Then the unique deep quad through $L$ distinct from $Q$ is equal to the unique quad $R$ through $L$ meeting $Q^{\prime}$.

Proof. The quad $R$ is singular or deep, but as $R \cap H$ contains two disjoint lines, namely $R \cap Q$ and $R \cap Q^{\prime}$, it must be deep.

Lemma 5.4 The spread $S$ is regular.

Proof. Let $L_{1}$ and $L_{2}$ be two disjoint lines of $S$. For every $i \in\{1,2\}$, let $R_{i}$ denote the unique deep quad through $L_{i}$ distinct from $Q$. Then $R_{1}$ and $R_{2}$ are disjoint. Now, every quad of $\Omega\left(R_{1}, R_{2}\right)$ is deep and intersects $Q$ in a line, necessarily belonging to $S$. 
The set of lines of $\widetilde{Q}$ obtained by intersecting $Q$ with the elements of $\Omega\left(R_{1}, R_{2}\right)$ is equal to $\left\{L_{1}, L_{2}\right\}^{\perp \perp}$, showing that $S$ is regular.

Now, let $\mathcal{Q}_{1}$ be the set of all deep quads intersecting $Q$ in a line. Let $Q^{\prime}$ be a given quad of $\mathcal{Q}_{1}$ and let $\mathcal{Q}_{2}$ denote the set of all deep quads intersecting $Q^{\prime}$ in a line. Then the following holds:

(1) $\mathcal{Q}_{i}, i \in\{1,2\}$, is a set of mutually disjoint quads;

(2) every quad of $\mathcal{Q}_{1}$ intersects every quad of $\mathcal{Q}_{2}$ in a line (by Lemma 5.3);

(3) if $Q \in \mathcal{Q}_{i}, i \in\{1,2\}$, then the set of lines of $\widetilde{Q}$ obtained by intersecting $Q$ with the elements of $\mathcal{Q}_{3-i}$ is a regular spread of $\widetilde{Q}$;

(4) if $Q_{1}$ and $Q_{2}$ are two distinct quads of $\mathcal{Q}_{i}, i \in\{1,2\}$, then $\Omega\left(Q_{1}, Q_{2}\right) \subseteq \mathcal{Q}_{i}$.

For every $i \in\{1,2\}$, let $X_{i}$ denote the set of points of $H\left(5, \mathbb{F}^{\prime}\right)$ corresponding to the quads of $\mathcal{Q}_{i}$, i.e. $X_{i}=\left\{x_{R} \mid R \in \mathcal{Q}_{i}\right\}$.

Lemma 5.5 For every $i \in\{1,2\}$, there exists a plane $\pi_{i}$ in $\mathrm{PG}\left(V_{6}^{\prime}\right)$ such that $\pi_{i} \cap H\left(5, \mathbb{F}^{\prime}\right)$ is a unital of $\pi_{i}$ equal to $X_{i}$. Moreover, if $\zeta$ denotes the Hermitian variety of $\mathrm{PG}\left(V_{6}^{\prime}\right)$ associated with $H\left(5, \mathbb{F}^{\prime}\right)$, then $\pi_{1}=\pi_{2}^{\zeta}$.

Proof. Let $u$ and $v$ be two distinct points of $X_{2}$ and let $Q_{u}$ and $Q_{v}$ denote the quads corresponding to $u$ and $v$, respectively. Since $Q_{u}$ and $Q_{v}$ are disjoint, the points $u$ and $v$ are noncollinear on $H\left(5, \mathbb{F}^{\prime}\right)$ and so the points and lines contained in $\{u, v\}^{\perp}$ define a generalized quadrangle $\mathcal{S}$ isomorphic to $H\left(3, \mathbb{F}^{\prime}\right)$. The points of $\mathcal{S}$ are obtained by intersecting $H\left(5, \mathbb{F}^{\prime}\right)$ with the 3-space $\alpha=(u v)^{\zeta}$. The quads meeting $Q_{u}$ and $Q_{v}$ are in bijective correspondence with the points of $\mathcal{S}$ and with the lines of $\widetilde{Q_{u}}$ (by considering the intersections with $Q_{u}$ ). So, there exists a bijective correspondence between the points of $\mathcal{S}$ and the lines of $\widetilde{Q_{u}}$. This bijective correspondence defines an isomorphism between $\mathcal{S} \cong H\left(3, \mathbb{F}^{\prime}\right)$ and the point-line dual of $\widetilde{Q_{u}}=Q(5, \mathbb{F})$. Now, the quads of $\mathcal{Q}_{1}$ meeting $Q_{u}$ and $Q_{v}$, intersect $Q_{u}$ in lines which determine a regular spread. By Proposition 3.7, there must exist a plane $\pi_{1}$ in $\alpha$ such that $\pi_{1} \cap H\left(5, \mathbb{F}^{\prime}\right)$ is a unital and equal to $X_{1}$. In a similar way, one proves that there exists a plane $\pi_{2}$ in $\operatorname{PG}\left(V_{6}^{\prime}\right)$ such that $\pi_{2} \cap H\left(5, \mathbb{F}^{\prime}\right)$ is a unital of $\pi_{2}$ equal to $X_{2}$. Since every quad of $\mathcal{Q}_{1}$ intersects every quad of $\mathcal{Q}_{2}$ in a line, we must have $\pi_{1}=\pi_{2}^{\zeta}$.

If we denote by $\mathcal{G}$ the union of all quads of $\mathcal{Q}_{1}$, then $\mathcal{G} \subseteq H$. The set $\mathcal{G}$ is also equal to the union of all quads of $\mathcal{Q}_{2}$. The point-line geometry $\widetilde{\mathcal{G}}$ induced on $\mathcal{G}$ by those lines of $\Delta$ that are contained in $\mathcal{G}$ is a glued near hexagon of type $Q(5, \mathbb{F}) \otimes Q(5, \mathbb{F})$. If $Q_{1}, Q_{2}$ and $Q_{3}$ are three mutually distinct quads of $\mathcal{Q}_{1}$ such that $Q_{3}$ does not belong to $\Omega\left(Q_{1}, Q_{2}\right)$, then $\left\langle\epsilon^{*}(\mathcal{G})\right\rangle=\left\langle\epsilon^{*}\left(Q_{1}\right), \epsilon^{*}\left(Q_{2}\right), \epsilon^{*}\left(Q_{3}\right)\right\rangle$ is 17-dimensional by Lemma 3.9. It follows from [6, Section 3] that $H$ belongs to one of the two classes of hyperplanes discussed in that paper. 


\section{A Some GAP code}

In this appendix, we list the GAP code we used to verify Lemma 2.6 in case the prime power $q$ belongs to $\{3,4,5,7,8\}$.

Suppose $\mathcal{U}$ is a unital in $\operatorname{PG}\left(2, q^{2}\right)$. The hyperbolic lines define a linear space $\mathcal{A}_{\mathcal{U}}$ on the set $\mathcal{U}$. Observe that $\mathrm{P} \Gamma \mathrm{L}\left(3, q^{2}\right)$ acts transitively on the set of hyperbolic lines. Let $L$ be a particular hyperbolic line of $\mathcal{U}$. Then $X:=L \cap \mathcal{U}$ is a line of $\mathcal{A}_{\mathcal{U}}$. Let $\mathcal{S}_{X}^{\prime}$ be the geometry as considered in Lemma 2.6. The following GAP code implements models for the geometries $\mathcal{A}_{\mathcal{U}}$ and $\mathcal{S}_{X}^{\prime}$. The point set of $\mathcal{A}_{\mathcal{U}}$ equals $\left\{1,2, \ldots, q^{3}+1\right\}$ and its line set is equal to lines1. The set line is a particular line of $\mathcal{A}_{\mathcal{U}}$. The point set of $\mathcal{S}_{X}^{\prime}$ is equal to points2 and its line set is equal to lines2.

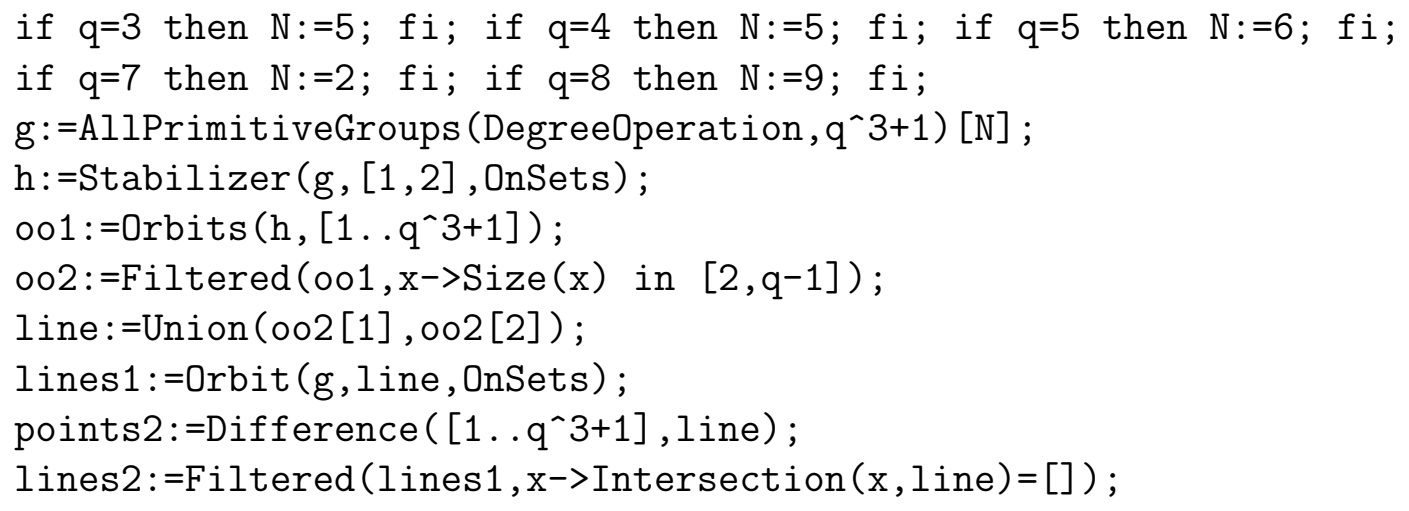

If $\mathrm{Y}$ is a set of points of $\mathcal{S}_{X}^{\prime}$, then Generate(Y) denotes the set of points of $\mathcal{S}_{X}^{\prime}$ generated by $\mathrm{Y}$.

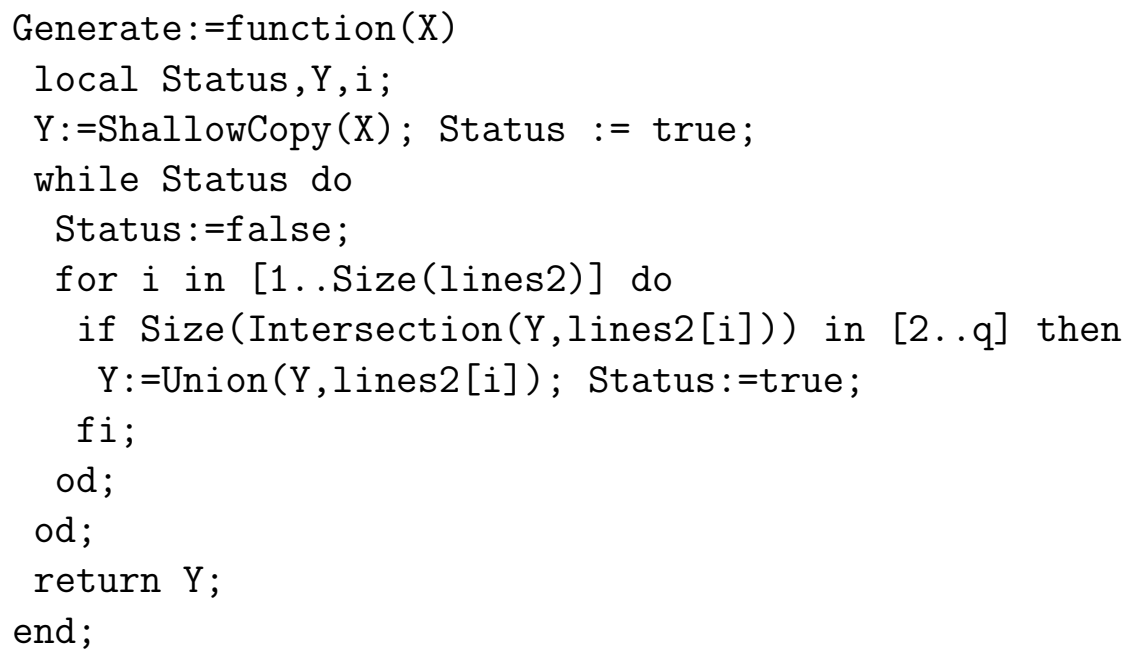

The stabilizer of $L$ (in $\mathrm{P} \Gamma \mathrm{L}\left(3, q^{2}\right)$ ) acts transitively on the points of $\mathcal{S}_{X}^{\prime}$. So, in order to verify Lemma 2.6, we may choose for $p_{3}$ a particular point of $\mathcal{S}_{X}^{\prime}$. Lemma 2.6 will then be valid if $L_{1} \cup L_{2}$ is a generating set of $\mathcal{S}_{X}^{\prime}$ for any pair $\left\{L_{1}, L_{2}\right\}$ of two distinct lines of $\mathcal{S}_{X}^{\prime}$ through $p_{3}$. We have verified this with the following GAP code. Lemma 2.6 is valid 
for the considered prime power $q \in\{3,4,5,7,8\}$ if the final value of Status is equal to true (which indeed turned out to be the case).

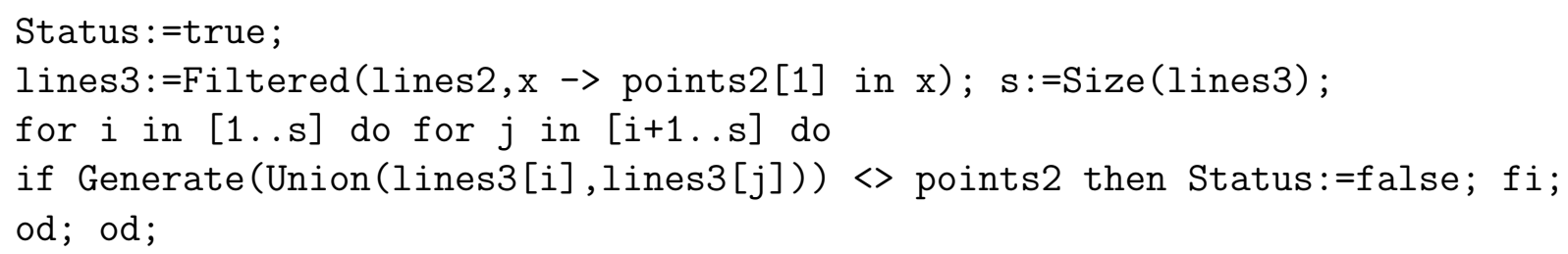

\section{References}

[1] R. J. Blok and A. E. Brouwer. Spanning point-line geometries in buildings of spherical type. J. Geom. 62 (1998), 26-35.

[2] I. Cardinali and B. De Bruyn. The structure of full polarized embeddings of symplectic and Hermitian dual polar spaces. Adv. Geom. 8 (2008), 111-137.

[3] B. N. Cooperstein. On the generation of dual polar spaces of unitary type over finite fields. European J. Combin. 18 (1997), 849-856.

[4] B. De Bruyn. On the Grassmann-embeddings of the Hermitian dual polar spaces. Linear Multilinear Algebra 56 (2008), 665-677.

[5] B. De Bruyn. Isometric full embeddings of $D W(2 n-1, q)$ into $D H\left(2 n-1, q^{2}\right)$. Finite Fields Appl. 14 (2008), 188-200.

[6] B. De Bruyn. On a class of hyperplanes of the symplectic and Hermitian dual polar spaces. Electron. J. Combin. 16 (2009), Research Paper 1, 20 pp.

[7] B. De Bruyn. Hyperplanes of $D W(5, \mathbb{K})$ containing a quad. Discrete Math. 313 (2013), 1237-1247.

[8] B. De Bruyn and A. Pasini. Generating symplectic and Hermitian dual polar spaces over arbitrary fields nonisomorphic to $\mathbb{F}_{2}$. Electron. J. Combin. 14 (2007), Research Paper 54, 17 pp.

[9] B. De Bruyn and H. Pralle. The exceptional hyperplanes of $D H(5,4)$. European J. Combin. 28 (2007), 1412-1418.

[10] B. De Bruyn and H. Pralle. The hyperplanes of DH(5, $\left.q^{2}\right)$. Forum Math. 20 (2008), 239-264.

[11] G. Faina and G. Korchmáros. A graphic characterization of Hermitian curves. Combinatorics '81 (Rome, 1981), 335-342, Ann. Discrete Math. 18, North-Holland, Amsterdam-New York, 1983.

[12] The GAP Group, GAP - Groups, Algorithms, and Programming, Version 4.4.12; 2008. (http://www.gap-system.org)

[13] C. Lefèvre-Percsy. Characterization of Hermitian curves. Arch. Math. (Basel) 39 (1982), 476-480. 
[14] S. E. Payne and J. A. Thas. Finite generalized quadrangles. Second edition. EMS Series of Lectures in Mathematics. European Mathematical Society (EMS), Zürich, 2009.

[15] M. A. Ronan. Embeddings and hyperplanes of discrete geometries. European J. Combin. 8 (1987), 179-185.

[16] S. Shad and E. Shult. The near $n$-gon geometries. Unpublished manuscript.

[17] E. Shult. On Veldkamp lines. Bull. Belg. Math. Soc. Simon Stevin 4 (1997), 299-316.

[18] M. Tallini Scafati. $\{k, n\}$-archi di un piano grafico finito, con particolare riguardo a quelli con due caratteri. Atti Accad. Naz. Lincei Rend. Cl. Sci. Fis. Mat. Natur. (8) 40 (1966), 812-818 and 1020-1025.

[19] M. Tallini Scafati. Caratterizzazione grafica delle forme hermitiane di un $S_{r, q}$. Rend. Mat. e Appl. (5) 26 (1967), 273-303. 